\title{
Turbulence analysis, modelling and computing using wavelets
}

\author{
M. Farge, N. K.-R. Kevlahan, V. Perrier \\ Laboratoire de Météorologie Dynamique du CNRS, \\ Ecole Normale Supérieure, \\ 24, rue Lhomond, 75231 Paris Cedex 5, France \\ K. SCHNEIDER \\ ICT, Universität Karlsruhe (TH), \\ Kaiserstrasse 12, 76128 Karlsruhe, Germany
}

\begin{abstract}
We have used wavelets and wavelet packets to analyse, model and compute turbulent flows. The theory and open questions encountered in turbulence are presented. The wavelet-based techniques that we have developed to study turbulence are explained and the main results are summarized.
\end{abstract}

\section{Keywords}

Turbulence, wavelets, wavelet packets, coherent structures, multifractals, singularities

\subsection{Introduction}

In this chapter we will summarize the ten years of research we have done to try to better understand, model and compute fully-developed turbulent flows using wavelets and wavelet packets. Fully-developed turbulence is a highly nonlinear regime (very large Reynolds number tending to infinity) and is distinct from the transition to turbulence (Reynolds number of order 100 or 1000). We have chosen to present a personal point of view concerning the current state of our understanding of fully-developed turbulence. It may not always coincide with the point of view of other researchers in this field because many issues are still undecided and highly controversial. This paper is a substantially revised and extended version of: Wavelets and Turbulence by Farge, Kevlahan, Perrier and Goirand which appeared in Proceedings of the IEEE, vol. 84, no. 4, April 1996, pp. 639-669.

After more than a century of turbulence study [156], no convincing theoretical explanation has produced a consensus among physicists (for a historical review of various theories of turbulence see [143], [141], [64]). In fact, a large number of ad hoc 'phenomenological' models exist that are widely 
used by fluid mechanicians to interpret experiments and to compute many industrial applications (in aeronautics, combustion, meteorology ...) where turbulence plays a role. For these models there is no need to suppose the universality of turbulence since they are not derived from first principles. They are compared with experiment, such as wind tunnel measurements, in order to tune the parameters necessary to match the model to the observations. This procedure is done case by case, for a given type of turbulent flow and for a given geometry of the internal or external boundaries. Actually, it is still not known whether fully-developed turbulence has the universal behaviour (independence of initial and boundary conditions) which is generally assumed in the limit of small scales. Already in 1979 [63] we expressed reservations about our understanding of turbulence and thought that we did not yet know the pertinent questions to ask in order to guide research in this field. Nearly twenty years of work on the subject have persuaded us that we have not yet identified the appropriate objects, by which we mean the structures and elementary interactions, from which it will be possible to construct a satisfactory theory of turbulence. Turbulent flows are chaotic, i.e. sensitive to initial conditions, therefore we are looking for a statistical theory. The classical averages used at present do not appear to be adequate.

In our opinion, our present ignorance of the elementary physical mechanisms at work in turbulent flows arises in part from the fact that we perform averages using point measurements and also because we think in terms of Fourier modes. This approach already been pointed out by Zabusky [185] when he wrote:

In the last decade we have experienced a conceptual shift in our view of turbulence. For flows with strong velocity shear ... or other organizing characteristics, many now feel that the spectral description has inhibited fundamental progress. The next "El Dorado" lies in the mathematical understanding of coherent structures in weakly dissipative fluids: the formation, evolution and interaction of meta-stable vortex-like solutions of nonlinear partial differential equations ...'

By using point measurements or the Fourier representation, we probably miss the point, because these classical methods ignore the presence of the coherent vortices that one observes in physical space and whose dynamic role seems essential. As Hans Liepmann, successor to Von Karman as director of the Aeronautical Laboratory of Caltech, has commented [126], [125], in turbulence research we are like the drunk man who has lost his keys in a dark alley, but who finds it easier to search for them under the street light. Everyone knows that turbulence has to do with vortex production and interaction. This is even embedded in the Latin etymology of the word 'turbulence': turba for crowd and turbo for vortex. Namely, a turbulent flow 
can be described as 'a crowd of vortices in nonlinear interaction'. However, because we do not have a good enough theoretical grasp of the structure of these vortices, on the mechanism of their production by nonlinear instabilities, and on their long-range collective dynamics, we prefer to forget about them and content ourselves with studying turbulence as far as possible from regions where vortices are produced. In particular, as far as possible from boundaries.

This approach has led turbulence research for the last fifty years to explore the unphysical academic case of statistically stationary, homogeneous and isotropic turbulence, which, under those hypotheses and assuming periodic boundary conditions, represents turbulent fields in terms of Fourier modes. To construct this theory one needs to suppose that the injection of energy is confined to the low wavenumbers, while the dissipation of energy is confined to the high wavenumbers. This assumption allows us to define an intermediate range of wavenumbers, called the inertial range, where the flow behaves in a conservative manner, which then enables us to predict the scaling of the inertial range energy spectrum. Unfortunately these hypotheses are incompatible with the local production of vorticity in boundary layers or shear layers, due to the duality between physical localization and spectral localization: if you have one you cannot have the other and viceversa (Heisenberg's uncertainty principle). The same remark holds for the dissipation of energy. Incidentally, we are convinced that this lack of physical soundness of the statistical theory proposed in 1941 by Kolmogorov, and strongly advocated by Batchelor, explains why G. I. Taylor had never been convinced by this redirection of turbulence research. In fact, as early as 1938 he had already recognized the importance of vortices in turbulence when he wrote [170]:

The fact that small quantities of very high frequency disturbances appear, and increase as the speed increases, seems to confirm the view frequently put forward by the author that the dissipation of energy is due chiefly to the formation of very small regions where the vorticity is very high.

To refocus turbulence research towards a more physical approach, we should take up the challenge proposed by Hans Liepmann [126] during a workshop we organized in February 1997 in Santa Barbara:

As long as we are not be able to predict the drag on a sphere or the pressure drop in a pipe from first principles (namely from continuous, Newtonian, incompressible assumptions, without any other complications), we will not have made it!

As astonishing as it may seem, these two very 'simple' and basic problems are still open and should be taken as a serious challenge. Our conviction is 
that the wavelet representation, because it keeps track of both position and scale, can help us to address these problems and improve our understanding of fully-developed turbulence.

As far as we know, we have been the first to introduce wavelets to analyze turbulence in two [73] and then three dimensions [68], to design orthogonal wavelet algorithms to solve PDEs [148], to use wavelet packets to extract coherent vortices out of turbulent flows [67], to solve the NavierStokes equations in a wavelet basis [85], [40], and to force turbulent flows using wavelets [74]. We are convinced that the wavelet transform, which decomposes the vorticity field onto a set of smooth functions with compact (or quasi-compact) support and thus permits a representation in both space and scale, seems to be an appropriate tool, not only for analyzing and interpreting experimental results, but also for attempting to construct a more satisfactory statistical theory and to define new numerical methods to compute fully-developed turbulent flows. Moreover, the unconditional approximation property of the wavelet representation may help us to compute high Reynolds number flows, to replace periodic boundary conditions by more physical ones, and to simulate the local production of vortices at the wall or in shear layers, while controlling the quality (local resolution and smoothness) of the approximation. This is the programme we will expose in this chapter. We will discuss the results we have obtained in the last ten years, but it is still very much work in progress and it will be a long time before its potential is confirmed or denied.

Our chapter is organized as follows. We first state the problem of turbulence and the main open questions. We then focus on how wavelets and wavelet packets can be used to answer these questions. We present fractal and multifractal analysis, turbulence analysis and turbulence modelling, and finally the use of wavelets to numerically solve the Navier-Stokes equation. In conclusion we present several perspectives, and point out where new methods need to be developed in order to improve our understanding of turbulence.

\subsection{Open questions in turbulence}

\subsubsection{Definitions}

Turbulence is a highly unstable state of fluid flows, where by fluids we mean continuously movable and deformable media. Liquids, gases and plasmas are considered to be fluids when the scale of observation is much larger than the molecular mean free path. Turbulence is characterized by the Reynolds number, which is the ratio of the nonlinear inertial forces, responsible for 
the flow instability, to the linear dissipative damping, which converts kinetic energy into thermal energy. We will focus on 'fully developed turbulence', namely the limit of very large Reynolds numbers, which corresponds to, either very large velocities (strong advection), and/or very small viscosity (weak dissipation, which tends to a constants as the Reynolds number tends to infinity), and/or very large turbulent scales. For flows encountered in hydraulics and naval engineering Reynolds numbers are of the order of $10^{2}$ to $10^{6}$, in aeronautics (engines, airplanes, shuttles) $10^{6}$ to $10^{8}$, in meteorology and oceanography $10^{8}$ to $10^{12}$, and in astrophysics larger than $10^{12}$.

While the dissipation term is optimally represented in Fourier space because Fourier modes diagonalize the Laplacian operator (for periodic boundary conditions), the nonlinear convective term is very complicated in Fourier space where it becomes a convolution, i.e. all Fourier modes are involved. As fully developed turbulence corresponds to flows where nonlinear convection is dominant, i.e. is larger than linear dissipation by a factor of the order of Reynolds number, it is obvious that the Fourier representation is inadequate for studying and computing flows in this large Reynolds limit. We need to find a mathematical tool to optimally solve the nonlinear convection term, in the same way as the Fourier transform is the most economical representation to solve the linear dissipation term for the rather unphysical case of periodic boundary conditions. Surprisingly, however, all classical methods in turbulence rely on the Fourier representation, which is inappropriate for the nonlinear convection term. For a review of these methods the best references are Monin and Yaglom [141] for the statistical theory of three-dimensional turbulence and Kraichnan and Montgomery [108] for the statistical theory of two-dimensional turbulence.

Turbulence remains an unsolved problem because our traditional conceptual and technical tools are inadequate. For instance, classical Hamiltonian mechanics describes steady states of conservative systems, but turbulent flows are non-stationary and dissipative. Classical dynamics only solves systems with a few degrees of freedom, while fully developed turbulent flows have a very large, perhaps even infinite, number of degrees of freedom. Classical statistical theories deal with closed reversible systems in thermal equilibrium, but turbulent flows are open irreversible systems out of thermal equilibrium. Classical mathematical methods solve linear differential equations, but cannot (apart from a very few cases) integrate analytically the nonlinear partial differential equations encountered in the study of turbulence. In fact, even the existence and uniqueness of solutions of the Navier-Stokes equations describing the fluid motions is an unsolved problem when nonlinear advection becomes dominant, i.e. in the fully de- 
veloped turbulent regime. We should mention here a recent mathematical result [34] which gives, using multi-scale (Paley-Littlewood) decomposition, a local existence and uniqueness theorem for Navier-Stokes equations in $\mathbb{R}^{3}$ if initial conditions are sufficiently oscillating (in a Besov norm sense). Some other mathematical attempts have been made using divergence free vector wavelets [75], [18], but in all cases these proofs are done in an unbounded space. However, physical fluid flows are bounded either internally or externally, and we still do not know the optimal functional space for describing real turbulent flows.

In summary, the theory of fully developed turbulence is in what we call a pre-scientific phase, because we do not yet have an equation, nor a set of equations, that could be used to efficiently compute turbulent flows. The incompressible Navier-Stokes equations, which are the fundamental equations of fluid mechanics, are not the right ones for turbulence because their computational complexity becomes intractable for large Reynolds number flows. However, in this limit it should then be possible, as it is done in statistical mechanics, to define averaged quantities which would be the appropriate variables to describe turbulence and then find the corresponding transport equations to compute the evolution of these new quantities. Likewise, the Navier-Stokes equations can be derived from the Boltzmann equation by considering appropriate limits (Knudsen and Mach numbers tending to zero [10], [11]) and appropriate averaging procedures to define new coarse-grained variables (velocity and pressure) and associated transport coefficients (viscosity and density). The turbulence equations should be derived as a further step in this hierarchy of embedded approximations, but this scientific program may be impaired by the possible non-universality of turbulence, which remains an essential question to address.

More precisely, it is easier to define the appropriate parameters to go from Boltzmann to Navier-Stokes than from Navier-Stokes to turbulence equations [138]. In the first case only a linear averaging procedure, namely coarse-graining, is needed, while in the second case we have to find an appropriate nonlinear procedure, namely some conditional averaging. For this we should first identify the dynamically active structures constituting turbulent flows, classify their elementary interactions and define the averaging procedures to construct appropriate statistical observables. Wavelet analysis is a good tool for exploring this conditional averaging and for seeking an atomic decomposition of phase space, defined in both space and scale. Tennekes and Lumley in 1972 [171] had already the intuition of such a phase-space decomposition when they proposed to consider a turbulent flow as a superposition of Gaussian-shaped wave packets, they were calling 'eddies'; but we know 
since Balian's theorem [9] that we cannot built orthogonal bases with such functions. This is why we propose to use instead wavelet or wavelet packet bases to study how phase-space 'atoms' exchange energy, or other important dynamical quantity, during the flow evolution and possibly combine to form phase-space 'molecules'.

We still hope that there will be enough universality in the behaviour of these phase-space 'atoms' so that we can find a general theory and a set of equations to describe their evolution, but this could well be an unrealistic goal. In addition, wavelets may supply new functional bases better adapted to represent and compute turbulent flows, i.e. to extract their elementary dynamical entities, perform the appropriate averages on them, and predict the evolution of these statistical quantities.

\subsubsection{Navier-Stokes equations}

The fundamental equations of the dynamics of an incompressible (constant density of fluid elements) and Newtonian (rate of strain proportional to velocity gradients) fluid are the Navier-Stokes equations:

$$
\begin{aligned}
\frac{\partial \boldsymbol{V}}{\partial t}+(\boldsymbol{V} \cdot \nabla) \boldsymbol{V}+\frac{1}{\rho} \nabla P & =\nu \nabla^{2} \boldsymbol{V}+\boldsymbol{F}, \\
\nabla \cdot \boldsymbol{V} & =0,
\end{aligned}
$$

plus initial and boundary conditions,

where $t$ is the time, $\boldsymbol{V}$ the velocity, $P$ the pressure, $\boldsymbol{F}$ the resultant of the external forces per unit of mass, $\rho$ a constant density and $\nu$ a constant kinematic viscosity.

The mathematical difficulty of the Navier-Stokes equations arises from the fact that the small parameter $\nu$, which tends to zero in the limit of infinite Reynolds numbers, i.e. for fully developed turbulent flows, appears in the term containing the highest-order derivative, namely the dissipation term $\nu \nabla^{2} \boldsymbol{V}$. Thus the character of the equations changes as $\nu$ tends to zero, since in this limit it is the nonlinear advection term $(\boldsymbol{V} \cdot \nabla) \boldsymbol{V}$ which dominates. This singular limit seems similar to the semi-classical limit of quantum mechanics when the Planck's constant tends to zero; incidentally Planck's constant has the same dimensions as kinematic viscosity. When $\nu=0$, i.e. for infinite Reynolds numbers, the Navier-Stokes equations are called Euler's equations.

One of the physical difficulties of the Navier-Stokes equations comes from the incompressibility condition, namely the divergence-free requirement imposed by equation (1.2), which implies that the speed of sound is infinite. In 
this case any local perturbation is instantaneously transmitted throughout the whole domain. This requirement seems too drastic and quite unphysical because the speed of sound is large in real flows but never infinite. In the future we may prefer to consider instead weakly compressible Navier-Stokes equations to simplify the computation of turbulent flows and represent their local behaviour more accurately. Moreover, on physical grounds Euler's equations are unrealistic because the limit $\nu=0$ contradicts the fluid hypothesis, which supposes that the system is locally close to thermodynamical equilibrium due to molecular collisions (which implies macroscopic dissipation).

Taking the curl of equations (1.1) and (1.2) gives the equation of vorticity $\boldsymbol{\omega}$, the curl of velocity,

$$
\frac{\partial \boldsymbol{\omega}}{\partial t}+(\boldsymbol{V} \cdot \nabla) \boldsymbol{\omega}=(\boldsymbol{\omega} \cdot \nabla) \boldsymbol{V}+\nu \nabla^{2} \boldsymbol{\omega}+\nabla \times \boldsymbol{F} .
$$

In three dimensions this equation shows that vortex tubes may be stretched by velocity gradients, a mechanism which has been proposed to explain the transfer of energy towards the smallest scales of the flow. In two dimensions the right-hand side becomes zero, because the vorticity is then a pseudoscalar $\boldsymbol{\omega}=(0,0, \omega)$ perpendicular to the velocity gradients. The vorticity, and its infinitely many moments, are therefore Lagrangian invariants of the flow (Helmholtz theorem). In this case there is no vortex stretching and energy cannot cascade towards the smallest scales, but tends to accumulate into the largest scales, the so called inverse energy cascade [107], [14], while enstrophy instead cascades towards the smallest scales where it accumulates.

\subsubsection{Statistical theories of turbulence}

The first statistical method to analyse turbulent flows was proposed in 1894 by Reynolds [157] who assumed that turbulent flows can be described by ensemble averages, without considering the details of each flow realization. He then decomposed the velocity field $\boldsymbol{V}(\boldsymbol{x})$ into a mean contribution $\bar{u}_{i}$ plus fluctuations $u_{i^{\prime}}$ and rewrote the Navier-Stokes equations to predict the evolution of $\bar{u}_{i}$, which gives the Reynolds equations

$$
\frac{\partial \bar{u}_{i}}{\partial t}+\bar{u}_{j} \frac{\partial \bar{u}_{i}}{\partial x_{j}}+\frac{1}{\rho} \frac{\partial \bar{P}}{\partial x_{i}}=\frac{\partial}{\partial x_{j}}\left(\nu \frac{\partial \bar{u}_{i}}{\partial x_{j}}-\overline{u_{i}^{\prime} u_{j}^{\prime}}\right)+\bar{F}_{i} .
$$

To solve the Reynolds equations one should compute the second order moment of the velocity fluctuations $\overline{u_{i}^{\prime} u_{j}^{\prime}}$, called the Reynolds stress tensor, which in fact depends on the third order moment $\overline{u_{i}^{\prime} u_{j}^{\prime} u_{k}^{\prime}}(i, j$, and $k$ are 
dummy indices), which depends on the fourth order moment, and so on ad infinitum. This is the closure problem: there are more unknowns than equations and to solve the hierarchy of Reynolds equations the traditional strategy is to introduce another equation, or system of Reynolds equations, chosen from some a priori phenomenological hypotheses, to close the set of equations.

For instance, to close the hierarchy of Reynolds equations, Prandtl introduced a characteristic scale for the velocity fluctuations, called the mixing length, which led him to rewrite the Reynolds stress tensor as a turbulent diffusion term. Following an hypothesis proposed by Boussinesq [29], and by analogy with molecular diffusion which smoothes velocity gradients for scales smaller than the molecular mean free path, Prandtl assumed that there exists a turbulent diffusion which regularizes the mean velocity gradients for scales smaller than the mixing length. Unfortunately this hypothesis is wrong because, contrary to molecular diffusion, which is decoupled from the large scale motions and can then be modelled by a linear operator (Laplacian) with an appropriate transport coefficient (viscosity), turbulent motions interact nonlinearly at all scales and there is no spectral gap to decouple large scale motions from small scale motions. This is a major obstacle faced by all turbulence models and the closure problem remains open. This is also the reason why renormalization group techniques [184] and nonlinear Galerkin numerical methods [132] have not yet lived up to their promises. An important direction of research is to find a new representation of turbulent flows in which there is a gap, decoupling motions out of equilibrium from well thermalized motions, which can then be modelled. Such a separation seems only possible with a nonlinear closure, based on conditional averages which depend on the local behaviour of each flow realization. Nonlinear wavelet or wavelet packet filters are good candidates for this (see section 5.2).

Taylor [169], under the influence of Wiener with whom he was in correspondence [17] since his famous paper on turbulent diffusion [168], proposed in 1935 characterizing turbulent fields by their correlation functions, in particular by the Fourier transform of their two-point correlation function which gives their energy spectrum. This relies on Wiener-Khinchin's theorem, which states that the Fourier transform of one realization of a stationary and ergodic random process in $\mathbb{R}^{n}$ is the same as the Fourier transform of the correlation function of this process. Twenty years before Einstein [56] had outlined the same method to characterize fluctuating data, but he was not followed at the time [183]. To simplify the computation of correlation functions, Taylor made the hypothesis of statistical homogeneity 
and isotropy of turbulent flows, supposing that the averages are invariant under both translation and rotation. In the thirties Gebelein proposed applying the probability theory of Kolmogorov to hydrodynamics, a method later developed by Kolmogorov himself and his student Obukhov [144], who published in 1941 three key papers on the statistical theory of fully developed turbulence. Kolmogorov [103], [104], [105] studied the way in which Navier-Stokes equations in three dimensions distribute energy among the different scales of the flow. This type of approach is common in statistical mechanics, but a difficulty arises here from the fact that turbulent flows are open thermodynamical systems, due to the injection of energy by external forces and its dissipation by viscous frictional forces. To resolve this difficulty Kolmogorov supposed that external forces act only on the largest scales while frictional forces act only on the smallest scales, which, in the limit of very large Reynolds numbers, leaves an intermediate range of scales, called the inertial range, in which energy is conserved and only transferred from large to small scales at a constant rate $\epsilon$ which is supposed to be constant. But this cascade of energy concerns ensemble averages and not individual flow realization; moreover, it is only phenomenological and has never been proved from first principles. Kolmogorov also supposed that turbulent flows are statistically homogeneous and isotropic; he also uses the fact that the skewness, namely the departure from Gaussianity of the velocity increment probability distribution, is constant, which implies that the flow is nonintermittent. These hypotheses lead him to the K41 model which predicts the following energy spectrum scaling, known as the $k^{-5 / 3}$ law

$$
E(k)=C \epsilon^{2 / 3} k^{-5 / 3}
$$

where $k$ is the modulus of the wavenumber averaged over directions, corresponding to the inverse of the scale, and $C$ is called Kolmogorov's constant.

Landau criticized Kolmogorov's hypothesis of a constant rate of energy transfer $\epsilon$ independent of the scale, arguing that the dissipation field should also be considered random. Following this remark Kolmogorov proposed to model the energy transfer as a multiplication process where only a fraction $\beta$ of energy is transferred from one scale to another. Assuming that the probability density of the dissipation field varies randomly in space and time with a log-normal law, this lead him to propose the K62 model which predicts the following energy spectrum scaling

$$
E(k)=C \epsilon^{\frac{2}{3}} k^{-\frac{5}{3}} \ln \left(\frac{k}{k_{I}}\right)^{\beta}
$$


where $k_{I}$ is the wavenumber at which energy is injected (inverse of the integral length scale).

For two-dimensional turbulence there is a statistical theory similar to Kolmogorov's theory developed by Batchelor [14] and Kraichnan [107]. This theory takes into account, in addition to the conservation of energy in the inertial range, the conservation of enstrophy (integral of vorticity squared), which is true only for the two-dimensional Euler equations. Making the same kind of hypotheses as Kolmogorov, they predicted a direct enstrophy cascade, from large to small scales, giving a $k^{-3}$ energy spectrum, and an inverse energy cascade, from small to large scales, giving a $k^{-5 / 3}$ energy spectrum. The problem is that the energy spectra obtained from numerical simulations are always steeper than the predicted $k^{-3}$. There is another more recent statistical theory proposed by Polyakov [153] which takes into account, in addition to the energy conservation, the conservation of infinitely many moments of vorticity in two dimensions, which led him to predict different scalings depending on the way energy is injected; thus, Polyakov's theory is not universal. In fact the same non-universal behaviour of twodimensional turbulence is also observed in numerical simulations [113].

Since the pioneering works of Onsager [146] and Joyce \& Montgomery [99], there are several statistical theories for decaying two-dimensional turbulence [161], [135], [162], [48], [163], [61] which are not based on ensemble averages nor Fourier representation. These theories, unlike those of Kraichnan's and Polyakov's, do not discard the spatial flow structure. For a recent review of these theories a good reference is [131]. Onsager's theory assumes that all vorticity is concentrated into a finite number of point vortices and predicts that there exist negative temperature states; more precisely it predicts that high energy states can be favoured compared to low energy states, contrary to classical statistical physics. These negative temperature states correspond to the clustering of same-sign vortices characteristic of the inverse energy cascade of two-dimensional turbulence. But the extension of Onsager's approach to describe continuous vorticity fields, involving infinite number of degrees of freedom and therefore infinite Liouville measure, leads to a highly singular limit which has been overcome only recently using large deviation probabilities and maximum entropy techniques. This new theory, due independently to Robert [162], [163] and Miller [135], predicts final stationary states (in the absence of external forces) characterized by a functional relation between coarse-grained vorticity and streamfunction. This relation is called the coherence function and it seems to be verified for strong mixing situations, such as two-dimensional shear layers or vortex merging [167]. 


\subsubsection{Coherent structures}

Since the beginning of turbulence research there has been, alongside the statistical approach based on ensemble averages, a tendency to analyze each flow realization separately. This leads to the recognition that turbulence contains coherent structures, even at very large Reynolds numbers [98]. Examples of coherent structures include the Karman vortices observed by Roshko in 1961 at a Reynolds number of $10^{7}$ [164], the horseshoe vortices observed in turbulent boundary layers and mixing layers [37], and the vorticity tubes (often called filaments) [43], [31] observed in statistically homogeneous flows. Coherent structures are defined as local condensations of the vorticity field which survive for times much longer than the eddy turnover time characteristic of the turbulent fluctuations.

The vorticity field is easy to visualize in numerical experiments, but very difficult to visualize in laboratory experiments; therefore, one usually observes the pressure field instead. Indeed, if we take the divergence of equation (1.1) we obtain

$$
2 \nabla^{2} P / \rho+\sigma^{2}-\omega^{2}=\nabla \cdot \boldsymbol{F}
$$

where $\sigma=\frac{1}{2}\left(\partial_{i} u_{j}+\partial_{j} u_{i}\right)$ is the rate of strain which controls dissipation. This equation shows that vorticity concentrations, corresponding to coherent structures, are sources of low pressure, while strained regions, corresponding to dissipation, are sources of high pressure. Couder et al. [43], [31] recently measured the histogram of the probability distribution function of pressure and shown that for the large negative pressures it has an exponential behaviour, while for the pressures around zero it has a Gaussian behaviour. In other words, the coherent structures, which are characterized by strong depressions, are responsible for the non-Gaussian behaviour of turbulent flows, which is consistent with observations made before by Van Atta and Antonia [175] from measurements of the spatial gradients of velocity. This has also been shown by Abry et al. [2] using wavelet techniques to separate the coherent structures from the background flow in a one-dimensional cut of pressure signal.

The mere existence of finite (and quite small) coherent structures [181] may invalidate the ergodic hypothesis, which is an essential ingredient of any statistical theory, necessary to replace ensemble averages by space averages. Then, according to Taylor's hypothesis, which requires that fluctuating velocities should be much smaller than the mean velocity, space averages can be replaced by time averages, which are easier to obtain in laboratory experiments. As far as we know, almost all existing laboratory results measuring 
the turbulence energy spectrum rely on Taylor's hypothesis. We are therefore sceptical of their validity when the coherent structures produce rare but intense velocity fluctuations. In this case, even though the rms of the velocity fluctuations remains small compared to the mean velocity, it is not clear that time and space averages can be interchanged.

Concerning numerical experiments, we interpret the energy spectrum, and its inertial range power-law form, as characteristic of the random processes responsible for turbulence. In practice, however, we analyze only one flow realization because in most simulations the correlation length is the of the order of the size of the computational periodic domain. In this case a powerlaw behaviour could be interpreted as indicating the presence of some quasisingular structures in the flow, and not as a proof of its random dynamics. This new point of view led Saffman [165] to interpret the energy power-law behaviour as resulting from the presence of vorticity fronts. Later Farge and Holschneider [69] proposed another interpretation based on the emergence of cusp-like coherent structures. In the limit of an infinite Reynolds number, these vorticity cusps will tend to point vortices, which correspond to the limit case of negative temperature states [33]. The wavelet transform, because it measures the local scaling of a field, is the appropriate tool for verifying these different interpretations in relating the power-law scaling of the energy spectrum to the geometry of coherent structures.

Today we still do not have a complete theory to explain the formation and persistence of coherent structures, and we shall have to content ourselves with a qualitative description of their behaviour. This is more evidence that we are still in a pre-scientific phase, having as yet only a limited grasp of the nature of turbulence. The new point of view is to consider that coherent structures are generic to turbulent flows, even at very high Reynolds numbers, and that they probably play an essential role in their intermittency. Indeed, several wind tunnel experiments [16], [4] have shown that the energy associated with the smallest scales of turbulent flows is not distributed densely in space and time. This has led various authors to conjecture that the support of the set on which dissipation occurs should be fractal [130], [80], or multifractal [147]. It is now thought, but not proven, that the time and space intermittency of turbulent flows is related to the presence of coherent structures [68]. This is still an open question and wavelet analysis seems to be one of the appropriate techniques to answer it.

The classical theory of turbulence is blind to the presence of coherent structures because they are advected by the flow in a homogeneous and isotropic random fashion, and hence they are lost by low-order moments. Moreover, the spatial support of coherent structures becomes smaller and 
smaller when Reynolds number increases, whereas in three-dimensional flows coherent structures (vorticity tubes often called filaments) become highly unstable [43] and therefore their temporal and spatial support may be very small. Consequently, the presence of coherent structures only affects the high-order velocity structure functions (defined as the high-order statistical moments of the velocity increments) which are most sensitive to rare and extreme events (large deviations). The high-order structure functions have been measured only recently [4], because their calculation requires very long data sequences. They do not follow Kolmogorov's theory which predicts a linear dependence of the scaling exponent of the velocity structure functions on their order. Van der Water [177] has observed that there are in fact two distinct nonlinear dependencies for odd and for even orders, which may be interpreted in terms of the multi-spiral model of Vassilicos [176].

It is important to provide statistical predictions based on coherent structure models. It is shown in [136] that a system of singular vortex elements in two dimensions and three dimensions possesses statistics that deviate from Gaussian. In that work it was shown that probability density functions (PDF's) of velocity derivatives are Lévy stable distributions with exponents different 2 (i.e. the PDF's are not Gaussian). The experimental evidence of similar findings is contained in the work of Goldburg and collaborators [172] in which the Cauchy distribution, predicted in [136] to be the consequence of $1 / r$ velocity decay of a vortex, is seen for the region of small velocity differences. The results of [136] also indicate that the tails of PDF's are determined by the structure of vortex cores.

In conclusion, we can say that the presence of coherent structures is probably responsible for the non-Gaussian statistics of fully developed turbulent flows. Due to the sensitivity to initial conditions of turbulent flows, any theory of turbulence should be statistical. But, before being able to construct a new statistical theory of turbulence, we need to find new types of averages able to preserve the information associated with coherent structures and therefore take into account the intermittency of turbulent flows. Wavelets can play a role there in separating the coherent (non-Gaussian) components from the incoherent (Gaussian) components of turbulent flows, in order to devise new conditional averages to replace the classical ensemble averages.

\subsection{Fractals and singularities}

\subsubsection{Introduction}

According to the K41 model turbulence in the inertial range has a power law energy spectrum (1.5), and thus does not have a characteristic length 
scale. Therefore turbulence in this range of length scales looks similar at any magnification and can be described as self-similar. According to experimental observations, however, turbulence is also characterized by quasi-singular structures such as vortices and is intermittent (quantities such as energy dissipation vary greatly in time and space). A quasi-singular structure is one that appears singular until the dissipation scale at which the smoothing effect of viscosity becomes important $\dagger$. In fact the theoretical $k^{-\frac{5}{3}}$ inertial range energy spectrum predicted by Kolmogorov's theory implies that some sort of quasi-singular distribution of velocity and vorticity must be present in turbulent flows [94], [137], [89]. This quasi-singular distribution could be the result of a set of quasi-singular structures (e.g. vortices), or due to a particular statistical distribution of structures (independently of their smoothness). One of the difficulties in turbulent flow analysis is how to disentangle these different contributions to the overall statistics.

It remains an open question whether this quasi-singular behaviour is due to the randomness of turbulent motions resulting from their chaotic dynamics or to the presence of localized quasi-singular structures resulting from an internal organization of the turbulent motions. Kolmogorov's theory is based on ensemble averages, but in using them we are unable to disentangle these two hypotheses. Ensemble averages should be replaced by an analysis of turbulence for each realization and be based on the local measurement and statistics of singularities for which we need effective ways of detecting and characterizing quasi-singularities in turbulent signals.

The types of possible singularities in the turbulent velocity or vorticity may be divided into two classes: cusps (i.e. non-oscillating singularities in which the function or one of its derivatives approaches infinity at a certain point, e.g. $1 / x$ ) and spirals (i.e. oscillating singularities in which the frequency of oscillation approaches infinity at a certain point, e.g. $\sin (1 / x)$ ). Figure 1.6 shows an example of a two-dimensional flow containing both cusps and a spiral. (A cut through the spiral is an oscillating singularity over a certain range of length scales.) Likewise the distribution of singularities in turbulence may also be divided into two classes: isolated (singularities at a finite number of points) and dense (singularities at an infinite number of points in a finite area). Dense distributions of singularities are called fractals and are characterized by one (monofractal) or more (multifractal) fractal dimensions. Figure 1.1(a) shows a typical fractal signal. Note that fractals may contain both cusp and spiral type singularities. Turbulence might contain both fractal and isolated distributions of singularities, and

$\dagger$ Note that for simplicity we shall use the terms 'singular' instead of 'quasi-singular' and 'infinite' instead of 'very large' throughout this section. 
Fig. 1.1. Different types and combinations of singularities. (a) A fractal signal with energy spectrum $E(k) \propto k^{-\frac{5}{3}}$. (b) A spiral with fractal noise (both noise and spiral have the same energy spectrum $\left.E(k) \propto k^{-\frac{5}{3}}\right)$.

spiral and cusp types of singularities. Figure 1.1(b) shows a spiral type singularity with fractal noise superimposed; both the noise and the spiral have the same energy spectrum.

This section is concerned with wavelet-based techniques for calculating quantities such as energy spectra, structure functions, singularity spectra and fractal dimensions. These subjects are connected by the fact that they all measure the local regularity of the signal (i.e. strength of singularities in the signal). For example, the slope of the usual Fourier energy spectrum of a signal containing only isolated cusp singularities is determined by the strongest singularity [188]. The advantage of the wavelet transform is that it is able to analyse locally the singular behaviour of a signal. One can then use this local information to construct statistics describing the distribution and type of singularities (e.g. multifractals), and define local or conditionally averaged versions of traditional diagnostics such as the energy spectrum and structure functions. We are primarily concerned with cusp type singularities (either isolated or fractal), although we also discuss methods for distinguishing between signals containing isolated spirals and purely fractal signals.

In subsection 1.3.2 we review the mathematical results on one of the key properties of wavelet transforms: their ability to detect and characterize singular structures. We then describe three related applications which rely on this property: calculation of local energy spectra, structure functions (subsection 1.3.3) and the singularity spectra which characterize multifractals (subsection 1.3.5). These wavelet methods generally require the assumption that the singularities of the signal are cusps. Because isolated spirals are 
likely to be present in turbulence it is essential to have a method of determining which sort of singularity a signal contains. In subsection 1.3.6 we review a different wavelet-based method for distinguishing between signals containing isolated spirals and purely fractal signals (the two types of signal most likely to be measured in a turbulent flow). Each section gives a practical review of the method and briefly summarizes some results that have been obtained for turbulence data. Formulating these techniques in terms of wavelet transforms brings out the connections between them as well as providing new information, and this point is emphasized throughout this section.

\subsubsection{Detection and characterization of singularities}

The most useful property of the wavelet transform is its ability to detect and accurately measure the strength (given by the Hölder exponent) of individual singularities in a signal. We will first give a precise definition of the Hölder exponent.

A function $f(x)$, such that

$$
f: \mathbb{R} \rightarrow \mathbb{R},
$$

is said to belong to the Hölder space $C^{\alpha}$ for $\alpha$ a positive non-integer if there exists a constant $C$ such that for each $x_{0}$, there exists a polynomial $P$ of order less than $\alpha$ such that

$$
\left|f(x)-P\left(x-x_{0}\right)\right| \leq C\left|x-x_{0}\right|^{\alpha} .
$$

$f$ is said to have the Hölder exponent $\alpha\left(x_{0}\right)$ at point $x_{0}$ if $\alpha\left(x_{0}\right)=\sup \{\theta>$ $\left.0 / f \in C^{\theta}\left(x_{0}\right)\right\}$. The exponent $\alpha\left(x_{0}\right)$ therefore measures the smoothness of the function $f(x)$ near $x_{0}$ : the larger $\alpha\left(x_{0}\right)$ is, the smoother or more regular the function $f(x)$ is near $x_{0}$, while the smaller $\alpha\left(x_{0}\right)$ is, the rougher or more singular the function is. If the Hölder exponent is less than one, there is an actual singularity of the function at $x_{0}$ (or a quasi-singular behaviour near $x_{0}$ over a certain range of length scales if one is measuring a physical quantity like velocity).

It is important to note that equation (1.9) does not hold for oscillating singularities because in this case the Hölder exponent increases by more than one when the function is integrated. This anomalous behaviour is due to the fact that there are an infinite number of accumulating oscillations in the neighbourhood of the singularity.

Consider the $L^{1}$ norm wavelet transform (which conserves the $L^{1}$ norm of 
a function)

$$
\tilde{f}_{1}(x, r)=\frac{1}{r} \int_{-\infty}^{\infty} f\left(x^{\prime}\right) \psi\left(\frac{x^{\prime}-x}{r}\right) \mathrm{d} x^{\prime} .
$$

The wavelet transform is thus a two-dimensional function in position $x$ and scale $r>0$. Mallat and Hwang [129] have shown that singularities in $f(x)$ produce a maximum in the modulus of the wavelet transform $\left|\tilde{f}_{1}(x, r)\right|$ and that following the position of a wavelet modulus maximum as $r \rightarrow 0$ gives the position $x_{0}$ of the singularity. Furthermore, each singularity has an associated 'influence cone' defined by

$$
\left|x-x_{0}\right| \leq C r
$$

and if the singularity is an isolated cusp then the wavelet transform modulus for all points within the influence cone is

$$
\left|\tilde{f}_{1}(x, r)\right| \leq A r^{\alpha\left(x_{0}\right)}
$$

provided that at least the first $n>\alpha\left(x_{0}\right)$ moments of the analysing wavelet $\psi(x)$ vanish, where the $n^{t h}$ moment is defined by the integral

$$
\int_{-\infty}^{+\infty} x^{n} \psi(x) \mathrm{d} x
$$

Equation (1.12) shows that the Hölder regularity $\alpha\left(x_{0}\right)$ can be found from the slope of the graph of $\log \left|\tilde{f}_{1}(x, r)\right|$ versus $\log r$ at a position $x$ satisfying inequality (1.11). When several singularities are present only the nonoverlapping parts of the cones associated with each singularity satisfy (1.12). Intuitively, it is the self-similar scaling property of the wavelet which allows the wavelet transform to measure the rate of self-similar narrowing with decreasing scale which characterizes the strength of a cusp singularity.

If the singularity is not isolated and there is only one zero-crossing of the wavelet transform near $x_{0}$, one can find the regularity in the left and right neighbourhoods of $x_{0}$ by measuring the decay of the wavelet modulus transform along maxima lines of the wavelet transform to the left and right of the influence cone of $x_{0}$.

In practice, such graphs of $\log \left|\tilde{f}_{1}(x, r)\right|$ versus $\log r$ contain oscillations superimposed on the power-law behaviour which can make it difficult to determine the slope at larger scales. Vergassola and Frisch [178] showed that these oscillations are necessarily present for any self-similar random process whether or not the signal is multifractal (the lacunarity of multifractal signals should also produce oscillations). These oscillations can be reduced by finding the average decay of the wavelet modulus along many lines in the 
influence cone, or by averaging the decay along vertical lines at many different points (e.g. one may be interested in the conditionally averaged scaling of points in regions of irrotational straining). Arnéodo, Bacry and Muzy [5] have suggested that the deviations from a strict power-law may be reduced by measuring the decay of the modulus of the wavelet transform along the line of maximum modulus within the influence cone.

The analysis of signals containing spiral singularities either isolated (e.g. $\left.\sin 1 /\left|x-x_{0}\right|\right)$ or fractal (e.g. the Riemann-Weierstrass function) is more complicated because the worst singular behaviour of a spiral singularity appears outside the cone of influence. In this case one measures the decay as $r \rightarrow 0$ of the modulus of the wavelet transform along the set of points which are general maxima below the cone of influence (i.e. maxima in both the position and scale directions). This gives an upper bound on the Hölder exponent, but in general one has to use lines of maximum modulus both inside and outside the cone of influence to fully determine the singular behaviour of an oscillating singularity.

Arnéodo, Bacry and Muzy [6] have recently carried out work defining two wavelet-based exponents that measure the strength of an oscillating singularity. They find that the faster the frequency increases, the more irregular its derivative. In general, oscillating behaviour appears in fractal objects that are self-similar under non-hyperbolic mappings, e.g. the Riemann-Weierstrass function or the Farey-tree partitioning of rationals.

\subsubsection{Energy spectra}

The Fourier energy spectrum has been one of the most popular techniques for turbulence analysis, indeed traditional turbulence theory was constructed in Fourier space [15]. The Fourier energy spectrum $E(k)$ of the real function $f(x)$ is defined by

$$
E(k)=\frac{1}{2 \pi}|\hat{f}(k)|^{2} \quad \text { for } \quad k \geq 0
$$

where $(\hat{\cdot})$ signifies Fourier transform. Note that when analysing turbulence velocity signals one usually ensemble averages the energy spectra from many realizations (or integral length-scales). This produces a much smoother curve. In traditional turbulence theory only the modulus of the Fourier transform is used (e.g. the energy spectrum) and thus the phase information is lost. This is probably a major weakness of the traditional way of analysing turbulence since it neglects any spatial organization of the turbulent velocity field. 
The wavelet transform extends the concept of energy spectrum so that one can define a local energy spectrum $\tilde{E}(x, k)$ using the $L^{2}$ norm wavelet transform (which conserves the $L^{2}$ norm of a function) rather than the $L^{1}$ norm used in subsection 1.3.2 (i.e. the wavelet transform is normalized by $1 / r^{\frac{1}{2}}$ rather than by $1 / r$ and the resulting function is designated by $\tilde{f}$ instead of $\tilde{f}_{1}$ )

$$
\tilde{E}(x, k)=\frac{1}{2 c_{\psi} k_{0}}\left|\tilde{f}\left(x, \frac{k_{0}}{k}\right)\right|^{2} \quad \text { for } \quad k \geq 0
$$

where $k_{0}$ is the peak wave number of the analysing wavelet $\psi$ and

$$
c_{\psi}=\int_{0}^{+\infty} \frac{|\hat{\psi}(k)|^{2}}{k} \mathrm{~d} k
$$

By measuring $\tilde{E}(x, k)$ at different places in a turbulent flow one might estimate what parts of the flow contribute most to the overall Fourier energy spectrum and how the energy spectrum depends on local flow conditions. For example, one can determine the type of energy spectrum contributed by coherent structures, such as isolated vortices, and the type of energy spectrum contributed by the unorganized part of the flow.

Since the wavelet transform analyses the flow into wavelets rather than sine waves it is possible that the mean wavelet energy spectrum may not always have the same slope as the Fourier energy spectrum. Perrier, Philipovitch and Basdevant [149] have shown, however, that the mean wavelet spectrum $\tilde{E}(k)$

$$
\tilde{E}(k)=\int_{0}^{+\infty} \tilde{E}(x, k) \mathrm{d} x
$$

gives the correct Fourier exponent for a power-law Fourier energy spectrum $E(k) \propto k^{-\beta}$ provided that the analysing wavelet has at least $n>(\beta-1) / 2$ vanishing moments. This condition is obviously the same as that for detecting singularities derived in the previous section since $\beta=1+2 \alpha$ for isolated cusps. Thus, the steeper the energy spectrum the more vanishing moments of the wavelet we need. The inertial range in turbulence has a power-law form. The ability to correctly characterize power-law energy spectra is therefore a very important property of the wavelet transform (which is of course related to its ability to detect and characterize singularities).

Note that if the singularities are all isolated cusps then the exponent of the Fourier energy spectrum is determined by the strongest singularity $\alpha$ of the signal

$$
E(k)=C k^{-2(\alpha+1)},
$$


where $C$ is a constant. If the singularities are spirals and/or are not isolated then the strongest singularity sets a lower bound on the exponent of the energy spectrum [188]

$$
E(k) \leq C k^{-2 \alpha} .
$$

The way the dense singularities accumulate can make the signal effectively more singular, decreasing the magnitude of the exponent of the energy spectrum by up to 2 . Because they are both controlled in the same way by singularities, the wavelet energy spectrum can be thought of as a sort of local Fourier transform.

The mean wavelet energy spectrum is a smoothed version of the Fourier energy spectrum. This can be seen from the following relation between the two spectra

$$
\tilde{E}(k)=\frac{1}{c_{\psi} k} \int_{0}^{+\infty} E(\omega)\left|\hat{\psi}\left(\frac{k_{0} \omega}{k}\right)\right|^{2} \mathrm{~d} \omega
$$

which shows that the mean wavelet spectrum is an average of the Fourier spectrum weighted by the square of the Fourier transform of the analysing wavelet shifted at wavenumber $k$. Note that the larger $k$ is, the larger the averaging interval. This property of the mean wavelet energy spectrum is particularly useful for turbulent flows. The Fourier energy spectrum of a single realization of a turbulent flow is too spiky to be useful, but one can measure a well-defined slope from the mean wavelet energy spectrum.

The Mexican hat wavelet

$$
\hat{\psi}(k)=k^{2} \exp \left(-k^{2} / 2\right)
$$

has only two vanishing moments and thus can correctly measure energy spectrum exponents up to $\beta<5$. Only the zeroth order moment of the Morlet wavelet

$$
\begin{aligned}
\hat{\psi}(k) & =\frac{1}{2 \pi} \exp \left(-\left(k-k_{\psi}\right)^{2} / 2\right) \quad \text { for } \quad k>0 \\
\hat{\psi}(k) & =0 \text { for } \quad k \leq 0
\end{aligned}
$$

is zero, but the higher $n^{\text {th }}$ order moments are very small $\left(\propto k_{\psi}^{n} \exp \left(-k_{\psi}^{2} / 2\right)\right)$ provided that $k_{\psi}$ is sufficiently large. Therefore the Morlet wavelet transform should give accurate estimates of the power-law exponent of the energy spectrum at least for approximately $\beta<7$ (if $k_{\psi}=6$ ).

Perrier and Basdevant [149] present a family of new wavelets with an infinite number of cancellations

$$
\hat{\pi}_{n}(k)=\alpha_{n} \exp \left(-\frac{1}{2}\left(k^{2}+\frac{1}{k^{2 n}}\right)\right), \quad n \geq 1,
$$


where $\alpha_{n}$ is chosen for normalization. The wavelets defined in (1.23) can therefore correctly measure any power-law energy spectrum. Furthermore, these wavelets can detect the difference between a power-law energy spectrum and a Gaussian energy spectrum $\left(E(k) \propto \exp \left(-\left(k / k_{0}\right)^{2}\right)\right)$. It is important to be able to determine at what wavenumber the power-law energy spectrum becomes exponential since this wavenumber defines the end of the inertial range of turbulence and the beginning of the dissipative range.

The first measurements of local energy spectra in turbulence was reported by Farge et al. [68] and Meneveau [133]. Meneveau used the discrete wavelet transform to measure local energy spectra in experimental and Direct Numerical Simulation (DNS) flows and found that the standard deviation of the local energy (a measure of the spatial fluctuation of energy) was approximately $100 \%$ throughout the inertial range. Meneveau also calculated the spatial fluctuation of $T(k)$ which measures the transfer of energy from all wavenumbers to wavenumber $k$. On average $T(k)$ is negative for the large scales and positive for the small scales, indicating that in three dimensional turbulence energy is transferred from the large scales to the small scales where it is eventually dissipated (in agreement with Richardson [159]'s cascade model of turbulence). Meneveau found, however, that at many places in the flow the energy cascade actually operates in the opposite direction, from small to large scales, indicating a local inverse energy cascade (also called back-scattering). This local spectral information, which links the physical and Fourier space views of turbulence, can only be obtained using the wavelet transform.

\subsubsection{Structure functions}

Another fundamental quantity in the classical theory of turbulence [103] is the $p^{t h}$ order structure function $S_{p}(r)$

$$
S_{p}(r)=\frac{1}{L} \int_{0}^{L}|f(x)-f(x+r)|^{p} \mathrm{~d} x,
$$

where $L \gg r$ is the length of the signal, and $L$ must be long enough so that $S_{p}(r)$ does not change if $L$ is increased (and thus the increments of $f$ should be stationary in $x$ ). The velocity signal of a turbulent flow varies in both space and time and between different realizations of the flow. Thus the integral in (1.24) should, in general, be replaced by a suitably defined ensemble average in order to calculate the structure function of turbulent velocities. To justify the use of space or time averages instead of ensemble averages (over different realizations of the flow), one supposes that the turbulent flow 
motions are ergodic, which is an unvalidated hypothesis and is probably wrong for two-dimensional turbulence [181]. If the energy spectrum exponent $\beta$ is in the range $1<\beta<3$ (as is usually the case for the inertial range of turbulence) the velocity increments are a stationary function even though the velocities themselves are not [45], this is a good reason to work with velocity increments rather than the velocities themselves since stationarity is necessary in order to justify estimating a quantity by averaging over space. The larger $p$ the more $S_{p}(r)$ is dominated by extreme events. Thus the $p^{t h}$ order structure function characterizes more and more extreme events as $p$ increases.

If $f(x)$ is self-similar then, just as in the case of the energy spectrum, the structure functions will have a power law dependence on the scale $r$

$$
S_{p}(r)=r^{\zeta(p)} .
$$

The first order structure function $\zeta(1)$ provides a measure of the smoothness of $f(x)$, and in fact $\zeta(1)$ is related to the box dimension $D_{F}$ of the graph of $f(x)$

$$
D_{F}=2-\zeta(1)
$$

where $D_{F}$ measures the space-fillingness of $f(x)$. The second order structure function is related to the energy spectrum by

$$
\beta=\zeta(2)+1 .
$$

The Kolmogorov theory [103] showed that the inertial range of turbulence has $\beta=5 / 3$, or equivalently that

$$
\zeta(p)=p / 3
$$

however recent experiments [4] have shown that the structure function exponents increase more slowly than linearly with $p$ for $p>5$, contradicting Kolmogorov's 1941 theory. The cause of this difference is generally thought to be the fact that the energy dissipation $\varepsilon(x)=(d u(x) / d x)^{2}$ is intermittent in space, i.e. it varies greatly from place to place.

The velocity increment $\Delta f(x, r)=|f(x)-f(x+r)|$ is equivalent to a wavelet transform with $\psi_{\Delta}(x)=\delta(x+1)-\delta(x)$. In fact Jaffard [95] has shown that the exponent $\eta(p)$ is defined by

$$
\tilde{S}_{p}(r)=\frac{1}{L} \int_{0}^{L}|\tilde{f}(x, r)|^{p} \mathrm{~d} x \sim r^{\eta(p)}
$$

is the same as $\zeta(p)$ provided $p>1$ and $\zeta(p)<p$, no matter what wavelet is used. The wavelet-based method of calculating the structure function unifies 
the analysis of structure functions with the calculation of energy spectra and the strength of local singularities. If one uses a wavelet with a sufficient number of vanishing moments, then the wavelet-based structure function $\tilde{S}_{p}(r)$ should also be more sensitive to larger $\alpha$ singularities since the equivalent wavelet for the structure function, $\psi_{\Delta}(x)$, has only one vanishing moment. By changing from an integral to a sum over wavelet maxima we circumvent the divergence of the integral for negative $p$ and thus one can extend the definition of structure functions to include negative $p$ 's (as in Arnéodo, Bacry and Muzy [5]'s Wavelet Maximum Modulus Method discussed in the following section).

The wavelet-based version of the structure function allows us to see directly how the structure function is determined by the singular behaviour of $f(x)$. From equation (1.12) the wavelet transform modulus is proportional to $r^{\alpha\left(x_{0}\right)}$ and thus, since $r \ll L$, the stronger singularities contribute most to the higher order structure functions and least to the lower order structure functions. In other words, the value of $\zeta(p)$ is determined mostly by the stronger singularities for large $p$ 's and mostly by the weaker singularities for small $p$ 's.

Davis, Marshak and Wiscombe [45] point out that the 'dissipation' of a discrete function $f_{j}, \varepsilon_{j}=\left|f_{j}-f_{j-1}\right|$, is in fact a measure. Because $\varepsilon_{j}$ is a measure, the generalized dimension $D(p)$ of $f(x)$ can be calculated from the exponent $K(p)$ of the structure function of $\varepsilon(x)$,

$$
D(p)=1-\frac{K(p)}{p-1} .
$$

The generalized dimension $D(p)$ is the dimension of the set containing the singularities that contribute most to the $p^{\text {th }}$ order structure function. Because $\varepsilon(x)$ is a homogeneous variable (for $1<\beta<3$ ) we have $0<\beta_{\varepsilon}\left(x_{0}\right)<1$ and thus $-1 / 2<\alpha\left(x_{0}\right)<0$. Because $\alpha\left(x_{0}\right)<0$ the dissipation contains actual singular behaviour (the dissipation tends to infinity).

In general terms the exponents $\zeta(p)$ characterize the homogeneity of the field, while the exponents $K(p)$ characterize the singularity of the field. One can learn a great deal about the behaviour of a signal from the variability of $\zeta(p)$ and $K(p)$ and from the value of the first structure function exponents $\zeta(1)$ and $K(1)$. This information is summarized in table 1.1.

Davis, Marshak and Wiscombe [45] introduced the 'mean multifractal plane' defined as the plane with coordinates given by the most informative exponents $0<\zeta(1)=2-D_{F}<1$ and $0<K^{\prime}(1)=1-D(1)<1$ (where $D_{F}$ is the fractal dimension and $D(1)$ is by definition the information dimension). The position of a particular flow or model on the mean multifractal plane is a 


\begin{tabular}{cc}
\hline \hline Value of structure function & Type of signal \\
\hline$\zeta(1)=0$ & stationary, $D_{F}=2$ \\
$\zeta(1)=1$ & noiseless, $D_{F}=1$ \\
$K^{\prime}(1)=0$ & weak variability \\
$K^{\prime}(1)=1$ & $\delta$-function \\
$\zeta(p)$ variable & non-stationary multifractal \\
$\zeta(p)$ constant & non-stationary monofractal \\
$K(p)$ variable & stationary multifractal \\
$K(p)$ constant & stationary monofractal \\
\hline \hline
\end{tabular}

Table 1.1. Properties of a signal from the behaviour of the exponents of its structure function $\zeta(p)$ and the structure function of the modulus of its derivative $K(p)$.

good indicator of its self-similar characteristics. The higher the flow's $K^{\prime}(1)$ component the more intermittent and multifractal it is, and the higher the flow's $\zeta(1)$ component, the smoother and less stationary it is. Experimental turbulent velocity fields lie in the centre of the mean multifractal plane. Turbulence models, however, tend to lie along the boundaries of the domain: purely multiplicative cascade models (such as $\delta$-functions) lie on the $K^{\prime}(1)$ axis and purely additive models (such as fractional Brownian motion) lie on the $\zeta(1)$ axis! This clearly indicates that the current turbulence models do not represent correctly the self-similar structure of turbulent flows.

\subsubsection{The singularity spectrum for multifractals}

In order to characterize a multifractal function it is necessary to calculate its singularity spectrum. The singularity spectrum $D(\alpha)$ may be defined as the Hausdorff (or 'fractal') dimension of the set of points with Hölder exponent a

$$
D(\alpha)=D_{F}\{x, \alpha(x)=\alpha\} .
$$

Note that this definition is equally valid for multifractal functions and measures. The singularity spectrum of a monofractal has only one point, e.g. the singularity spectrum of the fractional Brownian signal $B_{1 / 3}(x)$ which has a $k^{-\frac{5}{3}}$ energy spectrum is $D(\alpha=1 / 3)=1$ (the function $B_{1 / 3}(x)$ is singular everywhere with $\alpha=1 / 3$ ), while a the singularity spectrum of a multifractal is a curve.

Parisi \& Frisch [147] found a way of estimating the singularity spectrum 
from the Legendre transform of the structure function exponents $\zeta(p)$

$$
D(\alpha)=\inf _{p}(p \alpha-\zeta(p)+1)
$$

where, as explained in subsection 1.3.3, $\zeta(p)$ may be calculated using the wavelet transform.

Equation (1.32) can be derived heuristically by noticing that near a singularity of order $\alpha$

$$
|\tilde{f}(x, r)| \sim r^{\alpha},
$$

where we have used equation (1.12) and have written $\alpha=\alpha\left(x_{0}\right)$ for simplicity. Now, if the dimension of the points with singularity $\alpha$ is $D(\alpha)$ then there are about $r^{-D(\alpha)}$ 'boxes' (in this case wavelets) with the scaling (1.33) in each interval $r$, so that the total contribution to the integral (1.29) is $r^{\alpha p-D(\alpha)+1}$. To leading order the magnitude of the integral is given by the largest contribution so that

$$
\zeta(p)=\inf _{p}(\alpha p-D(\alpha)+1) .
$$

Since $\zeta(p)$ is concave, formula (1.32) can be obtained by an inverse Legendre transform.

Jaffard [95] proved mathematically, however, that structure function calculations of the singularity spectrum can, in general, only set an upper bound on $D(\alpha)$ and he gave some counterexamples where such calculations give completely misleading answers.

Arnéodo, Bacry and Muzy [5] have developed a method for calculating the singularity spectrum called the Wavelet Transform Modulus Maximum (WTMM) method. This method is closely related to the calculation of structure functions by wavelet transforms except that, instead of integrating (or summing in case of discretely defined functions) the wavelet transform over all positions, one only sums the wavelet transforms located at maxima, i.e.

$$
\tilde{\Sigma}_{p}(r)=\sum_{l \in L(r)}\left(\sup _{\left(x, r^{\prime}\right)}\left|\tilde{f}\left(x, r^{\prime}\right)\right|^{p}\right),
$$

where $l$ is a maxima line of the wavelet transform modulus on $[0, r]$ and $\sup _{\left(x, r^{\prime}\right)}$ means that the supremum is taken for $\left(x, r^{\prime}\right)$ on $l$ (so that $r^{\prime} \leq r$ ). The wavelets are in fact playing the role of 'generalized boxes' in a new form of the standard box-counting algorithm used to estimate fractal dimensions $D(\alpha)$. Summing only over the wavelet modulus maxima makes sense since, as Mallat \& Hwang [129] showed, most of the information in the wavelet transform is carried by the wavelet maxima lines. Furthermore, because one 


\begin{tabular}{cc}
\hline \hline Thermodynamic parameter & Multifractal parameter \\
\hline$T$ (temperature) & $p^{-1}$ \\
$Z$ (partition function) & $\tilde{\Sigma}_{p}(r)$ \\
$G$ (free energy) & $\tau(p)$ \\
$S$ (entropy) & $D(\alpha)$ \\
\hline \hline
\end{tabular}

Table 1.2. Analogies between statistical thermodynamics and the Wavelet Transform Modulus Maximum method for multifractals.

does not sum over places where the wavelet modulus is zero, $\tilde{\Sigma}_{p}(r)$ is also defined for $p<0$ as well as for $p \geq 0$. Note that the structure function methods are defined only for $p \geq 0$.

Arnéodo, Bacry and Muzy draw the analogy with statistical thermodynamics and interpret $\tilde{\Sigma}_{p}(r)$ as a 'partition function' (see table 1.2).

If $f(x)$ is a self-similar function then $\tilde{\Sigma}_{p}(r) \propto r^{\tau(p)}$ and the singularity spectrum can be found by calculating the Legendre transform

$$
D(\alpha)=\inf _{p}(p \alpha-\tau(p)) .
$$

To avoid technical problems associated with calculating the Legendre transform in (1.36) Arnéodo, Muzy and Bacry [5] recommend an alternative way of finding $D(\alpha)$ (see their paper for details).

Jaffard [95] proved mathematically that the WTMM method, unlike the structure function methods, gives the correct singularity spectrum for all $p$ provided it is slightly modified. Indeed a problem might arise if the wavelet modulus maxima are too close together; in that case the sum in an interval of width $r$ must be restricted to the largest maxima. Jaffard also shows that even the modified WTMM method fails if the function $f(x)$ contains too many oscillating singularities.

Arnéodo, Bacry and Muzy [5] find the relation between $\tau(p)$ and $\zeta(p)$ from their respective definitions in terms of $D(\alpha)$, but given the limitations of equation (1.32), it is perhaps better (and more intuitive) to find the connection directly through the structure functions. In terms of discrete signals, the wavelet transform-based calculation of the structure function (1.29) becomes

$$
\tilde{S}_{p}(r)=\frac{1}{N} \sum_{j=1, N}\left|\tilde{f}\left(x_{j}, r\right)\right|^{p}
$$

Each cone of influence of width $r$ must contain only maxima lines with the 
same scaling (since the scaling $r^{\alpha\left(x_{0}\right)}$ is the same for all points within the influence cone of point $x_{0}$ ) and if the function is everywhere singular all intervals of size $r$ must contain at least one maxima line. If one follows Jaffard [95]'s refinement to WTMM, and only counts one maximum for each interval of length $r$, then the number of terms in the sum must be proportional to $N / r$. Therefore, if the wavelet moduli are only summed over their maxima the structure function becomes

$$
\tilde{S}_{p}(r)=\frac{1}{N / r} \sum_{l \in L(r)}\left(\sup _{\left(x_{j}, r^{\prime}\right)}\left|\tilde{f}\left(x_{j}, r^{\prime}\right)\right|^{p}\right)=\frac{1}{N / r} \tilde{\Sigma}_{p}(r)
$$

We thus find that the relation between the structure function exponents $\zeta(p)$ and the WTMM 'free energy' exponents $\tau(p)$ is

$$
\zeta(p)=\tau(p)+1
$$

Note that equation (1.39) only holds if the function $f(x)$ has singularities everywhere and WTMM is modified by only counting one wavelet modulus maximum for each interval of length $r$.

Arnéodo, Bacry \& Muzy [5] applied the WTMM method to single point high Reynolds number (the Taylor scale based Reynolds number is $R_{\lambda}=$ 2720 ) velocity data obtained by Gagne [87] from the wind tunnel of ONERA at Modane. The self-similar inertial range follows the Kolmogorov $E(k) \sim$ $k^{-\frac{5}{3}}$ law for almost three decades. The WTMM analysis was carried out for this inertial range of scales on a section of data 100 integral (energy containing) scales long.

The histogram of singularities $\alpha\left(x_{0}\right)$ in the turbulence data was found to be quite wide and centred about the Kolmogorov value $\alpha=1 / 3$. Surprisingly, at some places in the flow $\alpha$ is negative which implies actual singular behaviour (velocity tending towards infinity). These negative $\alpha$ values may be spurious or may indicate the (rare) presence of strong vortices. The function $\tau(p)$ is convex which suggests that the regularity of the flow varies greatly from place to place. The singularity spectrum is peaked at the Kolmogorov value $\alpha_{\max }(p=0)=0.335 \pm 0.005$ with $D\left(\alpha_{\max }\right)=1.000 \pm 0.001$. This result indicates that the signal is fractal everywhere because the fractal support of $D\left(\alpha_{\max }\right)$ is equal to its topological dimension (i.e. the dimension of the signal, which is 1$)$. 


\subsubsection{Distinguishing between signals made up of isolated and dense singularities}

Although the inertial range of turbulence has a self-similar structure, not all self-similar functions are fractal; in fact one of the most physically plausible turbulence structures, the spiral vortex, can generate self-similar oscillating singularities with a non-trivial box-counting dimension (a technique to estimate the Hausdorff or fractal dimension). The conclusion drawn by Arnéodo, Bacry and Muzy [5] that turbulence is everywhere singular with a multifractal structure may be invalid if the turbulent velocity signal they analysed contains oscillating singularities. Because the WTMM method is only valid for signals that contain dense distributions of cusp type singularities, one should first try to determine whether a signal has isolated oscillating singularities before attempting to use the WTMM method. Unfortunately, the difference between signals containing singularities everywhere ('fractals') and signals containing a large number of isolated oscillating singularities (isolated 'spirals' in multi-dimensions or isolated 'chirps' in one dimension) is not obvious: both signals can have non-trivial box-counting dimensions.

Kevlahan and Vassilicos [101] developed two methods for distinguishing between isolated spiral and fractal signals based on the wavelet transform. (In fact their method only distinguishes between isolated and dense singularities, however isolated cusp singularities have a trivial box-counting dimension and thus can be distinguished from fractal signals on the basis of box-counting dimension alone.) The first method takes advantage of the fact that the singularities in a fractal are dense (there are singularities at an infinite number of points) whereas the singularities in an isolated spiral signal are isolated (the signal contains oscillating singularities only at the centres of spirals). If one averages the wavelet transforms of many realizations, or different data segments together, one can prove that the average wavelet transform modulus $\langle|\tilde{f}(x, r)|\rangle$ decays differently for the two types of singularity

$$
\text { as }\langle|\tilde{f}(x, r)|\rangle \propto N^{-1 / 2}\left|\tilde{f}\left(x_{0}, r\right)\right| \text { for fractal signals, }
$$

but,

$$
\text { as }\langle|\tilde{f}(x, r)|\rangle \propto\left|\tilde{f}\left(x_{0}, r\right)\right|, \quad r<L / N \quad \text { for spiral signals, }
$$

where $N$ is the number of segments averaged together and $L$ is the length of each segment. Thus, the average wavelet transform of the random phase fractal signal is $N^{-1 / 2}$ times a single realization, while that of the spiral signal does not depend on the number of realizations below a certain scale. 
The difference in the behaviour of $\langle|\tilde{f}(x, r)|\rangle$ is striking, and provides a diagnostic for determining whether a signal contains spiral-type singularities. This method was applied to the Gagne [87] turbulence data. The results were inconclusive, perhaps due to insufficient resolution near expected spiral scales or rarity of spiral vortices passing near the velocity probe.

The second method for distinguishing between isolated spiral and fractal singularities derives from the observation that the spatial fluctuation of wavelet energy $\tilde{E}(x, k)$ (measured by the standard deviation $\tilde{\sigma}(k)$ of $\tilde{E}(x, k)$ ) is independent of wavenumber for a random phase fractal signal, but increases with wavenumber for a spiral signal with the same energy spectrum. Analysis of the turbulent signal shows that $\tilde{\sigma}(k)$ increases with wavenumber (although at a slower rate than for the purely spiral test signal), indicating that turbulence probably contains some sort of isolated oscillating singularities. This conclusion should be borne in mind when interpreting the results of multifractal analyses of turbulence.

\subsection{Turbulence analysis}

\subsubsection{New diagnostics using wavelets}

It is impossible to define a local Fourier spectrum, because Fourier modes are non-local, but it is possible to define a local wavelet spectrum, since wavelets are localized functions. Actually, due to the inherent limitation of the uncertainty principle stating that there is a duality between spectral and spatial information, we should be aware that the spectral accuracy will be poor in the small scales and that the spatial accuracy will be poor in the large scales.

Since turbulent flows are either two-dimensional or three-dimensional, in the following section we will use the two-dimensional wavelet transform. Let us consider a two-dimensional scalar field $f(\boldsymbol{x})$ and a two-dimensional real isotropic wavelet $\psi(\boldsymbol{x})$. We generate the family $\psi_{\boldsymbol{x}, r}\left(\boldsymbol{x}^{\prime}\right)$ of wavelets, translated by position parameter $\boldsymbol{x} \in \mathbb{R}^{2}$, and dilated by scale parameter $r \in \mathbb{R}^{+}$, all having the same $L^{2}$ norm

$$
\psi_{\boldsymbol{x}, r}(\boldsymbol{x})=r^{-1} \psi\left(\frac{\boldsymbol{x}-\boldsymbol{x}^{\prime}}{r}\right) .
$$

The two-dimensional wavelet transform of $f(\boldsymbol{x})$ is

$$
\tilde{f}(\boldsymbol{x}, r)=\int_{\mathbb{R}^{2}} f\left(\boldsymbol{x}^{\prime}\right) \psi_{\boldsymbol{x}, r}\left(\boldsymbol{x}^{\prime}\right) d^{2} \boldsymbol{x}^{\prime}
$$


The local wavelet spectrum of $f(\boldsymbol{x})$ is defined as

$$
\tilde{E}(\boldsymbol{x}, r)=\frac{|\tilde{f}(\boldsymbol{x}, r)|^{2}}{r} .
$$

A characterization of the local 'activity' of $f(\boldsymbol{x})$ is given by its wavelet intermittency $\tilde{I}(\boldsymbol{x}, r)$, which measures local deviations from the mean spectrum of $f$ at every position $\boldsymbol{x}$ and scale $r$, defined as follows

$$
\tilde{I}(\boldsymbol{x}, r)=\frac{|\tilde{f}(\boldsymbol{x}, r)|^{2}}{\int_{\mathbb{R}^{2}}|\tilde{f}(\boldsymbol{x}, r)|^{2} d^{2} \boldsymbol{x}},
$$

Another measure of interest for turbulence is the wavelet Reynolds number $\tilde{R} e(\boldsymbol{x}, r)$, given by

$$
\tilde{R} e(\boldsymbol{x}, r)=\frac{\tilde{u}(\boldsymbol{x}, r) r}{\nu},
$$

where $r$ is the scale parameter, $\nu$ the kinetic viscosity of the fluid, and $\tilde{u}$ the root-mean-square (r.m.s.) value of the velocity field contribution at position $\boldsymbol{x}$ and scale $r$ defined as

$$
\tilde{u}(\boldsymbol{x}, r)=\left(\frac{1}{3 C_{\psi}} \sum_{i=1}^{3}\left|\tilde{\boldsymbol{u}}_{i}(\boldsymbol{x}, r)\right|^{2}\right)^{1 / 2},
$$

with the constant

$$
C_{\psi}=\int_{\mathbb{R}^{2}}|\hat{\psi}(\boldsymbol{k})|^{2} \frac{d^{2} \boldsymbol{k}}{|\boldsymbol{k}|^{2}}
$$

The expectation is that at large scales $r \sim L$, the wavelet Reynolds number should coincide with the usual large-scale Reynolds number $R e=u L / \nu$, where $u$ is the r.m.s. turbulent velocity and $L$ is an integral scale characterizing the flow. In the smallest scales (say $r \sim \eta$, where $\eta$ is the Kolmogorov scale of the flow which characterizes the high wavenumber limit of the inertial range where dissipation becomes significant), one expects this wavelet Reynolds number to be close to unity when averaged spatially. The question we want to address here is the variability of such a wavelet Reynolds number defined for space and scale: are there locations where such a Reynolds number at some small scale is much larger than in others, and how do such regions correlate with regions of small-scale activity in the flow? If so, then $\tilde{R} e(\boldsymbol{x}, r)$ gives an unambiguous measure of the nonlinear activity at small scales (or at any desired scale). Regions of high wavelet Reynolds number could then be interpreted as regions of strong nonlinearity.

Concerning the computation of energy and enstrophy transfers and fluxes, 
we should be aware that the results depend on the functional basis we consider. Indeed, due to Heisenberg's uncertainty principle, each representation measures different types of transfers and fluxes. In Fourier space one computes transfers between different independent wavenumber bands, which detect the modulations and resonances excited under the flow dynamics. In wavelet space one computes exchanges between different locations and different scales, which detect instead advections and scalings. But one should never forget that in wavelet space spatial resolution is bad in the large scales and spectral resolution is bad in the small scales, while, by duality, space resolution is good in the small scales and spectral resolution in good in the large scales. In an orthogonal wavelet basis, although all wavelets are independent in space and scale, they are not independent in wavenumber. In an orthogonal wavelet packet basis all wavelet packets are independent in space, scale and wavenumber, but their Fourier spectrum presents several peaks at distant wavenumbers and they are no longer local in wavenumber space; therefore wavelet packets are unable to precisely measure transfers between different wavenumber bands. This is the reason why a comparison between transfers computed in wavelets, in wavelet packets and in Fourier modes is misleading: these three diagnostics do not measure the same quantities!

\subsubsection{Two-dimensional turbulence analysis}

Unlike the velocity field, the vorticity field is invariant with respect to uniform rectilinear translations of the inertial frame (Galilean invariance). The dependence of streamlines and streaklines on the reference frame causes considerable difficulties in the study of fluid flows, particularly in observing and defining vortices. In fact due to its Galilean invariance, vorticity is the most suitable field for tracking the dynamics of turbulent flows, in both two and three dimensions. The vorticity field is directly accessible from numerical simulations, but is difficult to obtain from laboratory experiments. This is why we will now focus on vorticity fields obtained from direct numerical simulations (DNS) results. The drawback with DNS, i.e. the integration of Navier-Stokes equations without any ad hoc turbulence modelling, is that current supercomputers are only able to compute low Reynolds number flows (up to a few thousand).

Let us show an example of a wavelet analysis of an instantaneous vorticity field computed using the Navier-Stokes equations [151], [65]. We segment it into three regions using the Weiss criterion [180], [57], namely into rotational regions corresponding to the coherent structures, strongly strained regions corresponding to the shear layers surrounding the coherent structures, and 
weakly strained regions corresponding to the background flow made of vorticity filaments (these vorticity filaments encountered in two-dimensional turbulence are not the same dynamical objects as the vorticity tubes encountered in three-dimensional turbulence and often called filaments). We then decompose the vorticity field into a continuous wavelet representation using an isotropic (Hermite) wavelet to integrate in space the wavelet coefficients for each type of region. This decomposition is in fact a conditional statistical analysis because the energy spectrum is computed separately for each type of region.

The energy spectrum of the coherent structure regions tends to scale as $k^{-6}$, the sheared regions as $k^{-4}$ and the background regions as $k^{-3}$ (figure 1.2). We found [151], [66] that each region has energy throughout the inertial range and therefore there is no scale separation. This is why the Fourier representation cannot disentangle these different regions. The scaling of the coherent structures seems compatible with the cusp-like model proposed by Farge and Holschneider [69], the scaling of the shear layers seems compatible with the $k^{-4}$ spectrum predicted by Saffman [165] and only the scaling of the homogeneous background regions seems to verify the Batchelor-Kraichnan prediction of a $k^{-3}$ spectrum. From this analysis we confirm that there is no universal power-law scaling for two-dimensional turbulent flows; the slope of the Fourier energy spectrum varies with the density of coherent structures (their number per unit area in 2D and per unit volume in 3D), which depends on initial conditions and forcing (energy injection by external forces). We then conjecture that there may be a universal scaling for each region of the flow considered separately, but this has not yet been proven. Extensive wavelet analysis of very different types of turbulent flows would be necessary to check this conjecture.

The new approach we have proposed is to decompose turbulent flows into organized (and therefore inhomogeneous) components and random (and therefore homogeneous) components, which will have different scalings and different statistical properties [67]. We have observed that the former, corresponding to strong vorticity values, is non-Gaussian, while the latter, corresponding to weak vorticity values is Gaussian (see Section 1.6.4.3) and figure $1.11(\mathrm{~d})$ ). If this point of view is confirmed, then only conditional averaging will make sense. There is still some hope of finding a universal behaviour for each component taken separately, and we may then be able to design a new statistical theory of two-dimensional turbulence based on this property.

A key question, which remains open, is the following: is there a generic shape (namely a typical vorticity distribution) for coherent structures? The 
Fig. 1.2. Conditional wavelet spectra (this computation was done in collaboration with Thierry Philipovitch). (a) Vorticity field. In red: elliptic regions, dominated by rotation (antisymmetric part of the stress tens $\nabla \boldsymbol{V}$ ), which correspond to the coherent vortices. In blue: hyperbolic regions, dominated by strain (symmetric part of the stress tensor $\nabla \boldsymbol{V}$ ), which correspond to the incoherent background flow. (b) Coherent vortices where rotation dominates. (c) Shear layers where strain and strong velocity dominates. (d) Background flow where strain and weak velocity dominates. (e) Energy spectra. In black: Fourier energy spectrum, which tends to scale as $k^{-5}$. In dark blue: wavelet energy spectrum, which is a smooth approximation of the Fourier spectrum and tends to scale as $k^{-5}$. In red: wavelet energy spectrum of the coherent vortices, which tends to scale as $k^{-6}$. In green: wavelet energy spectrum of the shear layers, which tends to scale as $k^{-4}$. In light blue: wavelet energy spectrum of the background flow, which tends to scale as $k^{-3}$. 
answer to this question influences our analysis, in particular our interpretation in terms of scale, because the notion of scale is intrinsically linked to the generic shape we assume for the coherent structures. A prioris are as essential in statistical analysis as hypotheses are in modelling: we should state them clearly, otherwise our results will be nonsensical. For instance, without a definition of vortex shape the notion of vortex size and vortex circulation would be meaningless. A misunderstanding has persisted for years in the field of turbulence due to the identification of scale with the inverse wavenumber, which is true only if one assumes a wave-like shape for the vorticity field. Conversely, in other papers one encounters different implicit models of coherent structures (vortex patches, Gaussian vortices, or cusp-like vortices), which indeed condition our statistical analysis. Therefore one first needs a method to extract coherent structures out of turbulent flows in order to study them individually. The classical method consists of thresholding the vorticity field and identify as coherent vortices all regions where vorticity is larger than this threshold. However, the spectral information is then lost due to the discontinuity introduced by the threshold. We have proposed instead [72] two new methods based on the continuous wavelet representation, which preserves the regularity of the vorticity field and therefore its spectrum.

These methods depend on the choice of the analyzing wavelet and ideally we should use a wavelet which is a local solution of the linearized Navier-Stokes equations, namely a solution of the heat equation, such as any isotropic and smooth distribution of vorticity. This is why we use twodimensional Hermite wavelets (derivatives of the Gaussian), which are solutions of the heat equation. The higher the derivative, the better the cancellations and the more sensitive the wavelet will be to quasi-singular vortices, however its spatial selectivity will not be as good as for low order derivative wavelets. In the examples shown in this chapter (figures 1.2, 1.3 and 1.5) we use Marr's wavelet which is the Laplacian of the Gaussian.

The first method is to retain only the wavelet coefficients inside the influence cones (namely the spatial support of the wavelets) attached to the local maxima of the vorticity field corresponding to the centers of coherent structures; wavelet coefficients outside the influence cones are then discarded before reconstructing the vorticity field. The second method is to retain only the wavelet coefficients which are larger than a given threshold and to discard all other coefficients before reconstructing the vorticity field. We thus extract the coherent structures, and subtracting the original vorticity field gives us the background field. By computing the Fourier spectrum of these two fields we have confirmed our previous analysis: the energy spectrum of 
coherent structures tends to scale as $k^{-6}$ and that of the background field as $k^{-3}$ (figure 1.3). With our first method we can also extract just one coherent structure, analyze its shape, and compute its coherence function, namely the pointwise relation between vorticity and streamfunction, to check if it corresponds to the stationary states predicted by Montgomery's [99] or by Robert's [161],[162],[163] statistical theories. We are presently working in this direction, but have not yet published any result.

Another application of the wavelet representation in turbulence should be to design new types of forcing for numerical simulations. The method would consist of injecting energy and enstrophy at each time step, but only into the wavelet coefficients inside the influence cone corresponding to a given location. Depending on the type of forcing we want, we could either excite the same vortices or randomly select new vortices at each time step. Forcing is currently done in Fourier space and is rather unphysical, while waveletbased forcing could simulate the production of vorticity in boundary layers or mixing layers, which is a local process. This is another promising, but as yet untried, application of wavelet techniques for turbulent flow simulation.

\subsubsection{Three-dimensional turbulence analysis}

We have analysed different flow fields resulting from direct numerical simulations of three-dimensional turbulent flows [68], using the complex-valued Morlet wavelet, which plays the role of a numerical polarizer due to its angular selectivity, and whose complex modulus directly measures the energy density. We have first studied the temperature, velocity and pressure fields of a channel flow near the wall and have used the wavelet intermittency to pinpoint the regions of the flow dominated by strong nonlinear dynamics. It appears that the most intermittent regions are correlated with those of large vertical velocity, corresponding to ejections from the boundary layer. We have found that temperature behaves as a passive scalar almost everywhere, except in these very localized ejection regions. We have also observed that there is no return to isotropy in the small scales, contradicting one of the hypotheses of the statistical theory of turbulence, which supposes that turbulent flows become homogeneous and isotropic at small scales.

We have then analyzed the vorticity, velocity and a passive scalar in a temporal mixing layer after the mixing transition. We have found that wavelet intermittency is very strong, up to 120 , in the collapsing regions where the ribs (streamwise vorticity tubes produced by a three-dimensional instability) are stretched and engulfed into the primary spanwise vortex (produced by a two-dimensional Kelvin-Helmholtz instability). On the other hand, the 
Fig. 1.3. Wavelet packet compression of vorticity. (a) The uncompressed vorticity field and its Fourier spectrum which scales as $k^{-5}$. (b) The vorticity field reconstructed from the $5 \%$ strongest wavelet packet coefficients, which contains $89 \%$ of the total enstrophy, and its Fourier energy spectrum which scales as $k^{-6}$. (c) The vorticity field reconstructed from rest of the flow (95\% of wavelet packet coefficients) which contain $11 \%$ of the total enstrophy, and its Fourier energy spectrum which scales as $k^{-3}$. 
wavelet intermittency in the braids, i.e. outside the spanwise vortex, remains very low, not exceeding 5 . We have also noticed a return to isotropy in the small scales. From the local spectrum of the vertical vorticity we have observed that the collapsing regions have a spectral slope much shallower than the one of the braid regions; this departure from the space average wavelet spectrum increases with the scale and confirms the strong intermittency of the mixing layer. If we extrapolate the observed slopes, we conjecture that intermittency should increase with Reynolds number. We have then visualized the iso-surfaces of the wavelet Reynolds number, which can be interpreted as surfaces of iso-nonlinearity in the flow. The peaks on these iso-surfaces, which are associated with the most unstable regions, are located in the primary vortex core; this confirms our previous conclusions concerning the concentration of small-scale nonlinear activity there, due to the stretching of the ribs rolled around the primary vortex. We have also shown that the Kolmogorov scale, corresponding to the iso-surface $\operatorname{Re}(\boldsymbol{x}, r) \simeq 1$ where linear dissipation balances nonlinear advection, varies with location, being at much smaller scale in the vortex core than in the braids, with a scale variation of four octaves. This means that there should be some (spatially localized) dissipation for scales belonging to the inertial range. This observation contradicts Kolmogorov's hypothesis of non-dissipative energy transfers in the inertial range; but is in agreement with Castaing's theory of turbulence [35], [36], with Frisch and Vergassola[81]'s multi-fractal model and with Benzi et al's [20] extended self-similar model, which assume a weak dissipation in the inertial range.

For shear flows, such as the channel flow or the mixing layer we have studied, there is a clear correlation between large-scale events and small-scale activity, due to the presence of coherent structures. Wavelet analysis has been an essential tool for identifying them as phase-space regions correlated in both space and scale, where intermittency increases with scale [68]. We conjecture that for large Reynolds numbers these regions may become more and more localized and very intense in small-scale enstrophy. Therefore they are susceptible to develop singularities at very large Reynolds numbers. For the mixing layer these quasi-singular regions correspond to collapsing events, where the ribs are stretched and accumulated inside the primary vortex core, while for the channel flow these regions correspond to the tip of the horseshoe vortices ejected from the wall boundary layer. According to the Cafarelli-Kohn-Nirenberg theorem [32], singularities, if they exist, should be at most a set of Hausdorff measure one in space-time for any Reynolds numbers. Therefore if we want to look for quasi-singularities in three-dimensional turbulent flows we would be better of using a space-time 
continuous wavelet transform, whose theory is being initiated by DuvalDestin and Murenzi [55], but has not yet been sufficiently developed.

\subsection{Turbulence modelling}

We will now reconsider the closure problem mentioned in subsection 1.2.3, taking advantage of the new observations we have made of turbulent flows, and in particular the dynamical role of coherent structures, using the wavelet analysis.

\subsubsection{Two-dimensional turbulence modelling}

To compute turbulent flows we must separate the active components, responsible for their chaotic behaviour (namely sensitivity to initial conditions), from the passive components, which are advected by the velocity field resulting from the overall coherent structure motion. The active components are not in thermal equilibrium, while the passive components are well thermalized. Therefore the active components should be computed explicitly, while the passive components can be modelled by some ad hoc parameterization.

Classical numerical techniques (Galerkin methods [91], Large Eddy Simulation [117], [158], [119] and Nonlinear Galerkin methods [132]) assume that the active components are the low-wavenumber Fourier modes, or the scales resolved by the computational grid, while the passive components are the high-wavenumber Fourier modes, or the sub-grid scales. This scale separability of the turbulent dynamics is assumed to be true in both two and three dimensions.

We have shown [182] that a compression in the wavelet packet representation extracts the coherent structures out of the background flow, while the same amount of compression done in the adapted local cosine (Malvar) representation, which is a type of windowed Fourier basis, does not have this property (figure 1.4). Indeed, the more you compress in Fourier or windowed Fourier representations, the more you smooth the coherent structures, and consequently lose their enstrophy, destroy their phase information, and introduce parasitic wiggles in the background. Indeed, the more you compress the larger the effect of the analysing function. Therefore wavelets and wavelet packets, being localized functions, tend to separate coherent structures from the background flow (figure 1.4a), while Fourier and windowed Fourier, being non-localized functions, tend to smear coherent structures into the background flow (figure 1.4b). 
Fig. 1.4. Comparison between wavelet packet and adapted local cosine compression (this computation was done in collaboration with Echeyde Cubillo). (a) The uncompressed vorticity field. (b) The vorticity field reconstructed from the 70 strongest wavelet packet coefficients, which contain $90 \%$ of the enstrophy. (c) The vorticity field reconstructed from the 425 strongest adapted local cosine coefficients, which contain $90 \%$ of the total enstrophy. (d) Enstrophy contained in the retained coefficients versus their number. We observe, for instance, that 70 wavelet packet coefficients retain $90 \%$ of the total enstrophy, while 70 adapted local cosine coefficients retain only $50 \%$ of the total enstrophy. 
We have shown [67], using nonlinear wavelet packet compression, that there is no scale separability in two-dimensional turbulence. To prove this we have computed the time evolution of a two-dimensional turbulent flow which we use as our high-resolution reference flow. We have then compressed the initial vorticity field in two ways: either by retaining only the lower wavenumber Fourier modes, or by selecting the strongest (in $L^{2}$-norm) wavelet packet coefficients. We found that for a compression ratio of 200 the wavelet packet representation preserves, in a statistical sense (namely the energy spectrum is well predicted), the reference flow evolution while the Fourier representation leads to a statistically different solution. This conclusion is not surprising, considering the existence of an inverse energy cascade in two-dimensional turbulence, which implies that the highwavenumber Fourier modes remain active and affect the evolution of the low-wavenumber modes. The implication of this behaviour has not been implemented in turbulence models, because there were not yet any alternative method to replace grid-point and Fourier representations.

In the same paper [67] we showed that there is a possible separability between active modes, namely the coherent structures corresponding to the strong wavelet packet coefficients, and passive modes, namely the vorticity filaments of the background flow corresponding to the weak wavelet packet coefficients. Both components are multi-scale, which is why the Fourier representation is not able to disentangle them and a fortiori to model them. According to Weiss analysis [180] the coherent structures correspond to elliptic regions (nearby fluid trajectories remain nearby) where rotation $\omega^{2}$ dominates strain $\sigma^{2}$, while the background flow corresponds to hyperbolic regions (two nearby fluid trajectories separate exponentially) where strain $\sigma^{2}$ dominates rotation $\omega^{2}$. In the elliptic regions the local Reynolds number $\tilde{R} e(\boldsymbol{x}, r)$ is larger than one, while in the hyperbolic regions it is smaller than one, which indicates that the background flow is actually laminar (figure 1.5). Coherent structures are local quasi-stationary solutions of NavierStokes equations. The probability distribution of the velocity field associated to the coherent structures is out of thermal equilibrium and varies depending on their configuration in space. On the contrary the background flow has already thermalized due to the very strong mixing resulting from the straining imposed by the coherent structures. Therefore the probability distribution of the velocity field of the background flow is stationary and does no more depend on the spatial configuration of the coherent structures. We should then be able to model this background flow by an ad hoc stochastic process having the same enstrophy and the same statistics, in particular the same spectral slope, whereas the coherent structures should be explic- 
itly computed in phase-space. A possible direction would be to construct a wavelet or wave packet frame (namely a quasi-orthogonal basis) made of local solutions of the linearized Navier-Stokes equations (namely any isotropic smooth function). We do not yet know neither to construct it, nor to compute Navier-Stokes equations in it, but preliminary steps in this direction will be discussed in section 1.6.

We have also shown [100] that the presence of coherent structures inhibits the nonlinear instability of the background flow, namely the formation of new coherent structures. Using the wavelet packet representation to extract the coherent structures we then computed the evolution of the remaining background flow, in the absence of coherent structures, and observed the emergence of new ones out of it (figure 1.6). Actually when coherent structures are present, they impose a strain on the background flow, which then inhibits the formation of new coherent structures, and therefore there is no energy or enstrophy backscatter from the incoherent to the coherent components of two-dimensional flows. The next step to validate this observation will be to compute the different transfers between coherent and incoherent components of the flow (namely from coherent structures to coherent structures, from coherent structures to background, from background to coherent structures and from background to background) and check that there is no transfer from background to coherent structures. If this is confirmed, there will be a possible wavelet separability between the coherent and incoherent flow components and we may then be able to propose new parameterizations based on this gap.

\subsubsection{Three-dimensional turbulence modelling}

The assumption that the high-wavenumber Fourier modes are slaved to the active low-wavenumber Fourier modes, is probably also wrong for threedimensional turbulence due to the recent evidence of energy backscattering [49], [48], [50], [114], [152], i.e. inverse energy transfer from small to large scales, resulting from the presence of organized structures which locally interact and transfer energy to larger scales. We should take this observation with caution knowing that the amount of backscattering observed depends sensitively on the sharpness of the spectral filter used. There are two other reasons to explain why this assumption is not valid and should be revised.

The first reason comes from the fact that we do not have any universal theory of turbulence aside from the statistical theory which deals with homogeneous and isotropic ensemble averages, while a numerical simulation computes one flow realization at the time (at the highest resolution possible 
Fig. 1.5. Wavelet Reynolds number (this computation was done in collaboration with Thierry Philipovitch). (a) Velocity field computed with resolution $128^{2}$ ( $\Delta x=1$ unit length between two grid-points). (b) Wavelet Reynolds number at scale $64 \Delta x$, which fluctuates between 148 and 2700 with a mean value of 1713 . (c) Wavelet Reynolds number at scale $20 \Delta x$, which fluctuates between 31 and 578 with a mean value of 365 . (d) Wavelet Reynolds number at scale $8 \Delta x$, which fluctuates between 1 and 27 with a mean value of 17 . (e) Wavelet Reynolds number at scale $2 \Delta x$, which fluctuates between 0 and 3 with a mean value of 2 . 
Fig. 1.6. Dynamical analysis of coherent structures and incoherent background flow. (a) Total vorticity at $t=30$ computed with a resolution $1024^{2}$. (b) Vorticity corresponding to the coherent vortices alone at $t=30$. They are made up of 31 strong wavelet packet coefficients which contain $83 \%$ of the total enstrophy. (c) Energy spectra at $t=30$. In green: the total energy spectrum. In red: the coherent vortices energy spectrum. In blue: the filament energy spectrum. (d) Vorticity corresponding to the filaments alone at $t=30$. They are made up of 1048545 weak wavelet packet coefficients which contain $17 \%$ of the total enstrophy. (e) Integration of the total vorticity until $t=120$. (f) Integration of the coherent vortices alone until $t=120$. (g) Energy spectra at $t=120$. In green: the total energy spectrum. In red: the coherent vortices energy spectrum. In blue: the filament energy spectrum. (h) Integration of the filaments alone until $t=120$. 
with present supercomputers) and not ensemble averages (which will require too many computations of the same turbulent flow). Actually each flow realization is, unlike an ensemble average, highly inhomogeneous due to the presence of coherent structures. As we have shown in performing wavelet analyses of two and three dimensional turbulent flows, coherent structures are multi-scale and, through their mutual nonlinear interactions, are responsible for inverse energy transfers. If the computational grid is too coarse, its resolution is insufficient to accurately compute these transfers. Likewise sub-grid-scale parameterization is only able to model direct transfers (from resolved to unresolved scales) and inverse transfers (from unresolved to resolved scales) in a statistical sense, assuming homogeneity, but not for the given inhomogeneous flow realization one computes. In fact backscattering is a major unresolved drawback of current numerical methods, which will last as long as they will be unable to separate the coherent structures from the background flow and take into account the parameterization of homogeneous turbulent components separately from the inhomogeneous components.

The second reason comes from the fact that our current numerical methods are defined, either in grid-point, finite element or Fourier representation, and are unable to compute multi-scale objects with a small number of coefficients. This would be possible using either adapted multi-grid or wavelet numerical methods. Multi-grid techniques were proposed 20 years ago by Achi Brandt [30] for solving elliptic problems, such as the diffusion equation; they were then adapted to quasi-stationary problems, but do not seem yet optimal to solve time-dependent problems. Actually the multi-grid approach is very similar to a wavelet approach using a Haar wavelet, which is very well localized in physical space and corresponds to a set of embedded grids, but which is too de-localized in spectral space and tends to produce large errors in the higher order derivatives of the solution. As far as we know, locally refined multi-grid techniques have been tried for the Navier-Stokes equations, but not yet in the turbulent regime.

One possible approach is to use the wavelet Reynolds number to split the Navier-Stokes equations at each time step into advection and diffusion operators, which will be solved separately using the most appropriate numerical method and turbulence parameterization for each operator. Namely, the advection term is computed only where $\tilde{R} e(\boldsymbol{x}, r)>1$, and the diffusion term where $\tilde{R} e(\boldsymbol{x}, r)<1$. This method makes sense only if the flow is computed either in a multi-grid or in a wavelet representation section 1.6. We could, for instance, build an appropriate wavelet frame (namely a quasi-orthogonal basis) made of local solutions of the linearized Navier-Stokes equations, 
which could be any isotropic smooth function such as a circular Gaussian vortex (e.g. the Burger's vortex).

Actually, as we have already said, the Navier-Stokes equations are computationally intractable for the large Reynolds number limit which corresponds to fully developed turbulent flows. Although the use of wavelets may improve current numerical methods of solving the Navier-Stokes equations (see section 1.6), a more promising direction may be to look for a new set of equations specific to the turbulent regime. Such equations would be written in terms of a small number of new variables corresponding to the degrees of freedom attached to the coherent structures. As a consequence they may break some of the symmetries of Navier-Stokes, in particular its translational invariance. This is analogous to the way in which Boltzmann's equation, describing the macroscopic level, breaks the time reversibility of Newton's equation, describing the microscopic level. For modelling turbulent flows we ought to go one step further in this hierarchy of embedded equations and define a new 'organized' level emerging out of the thermalized background flow.

\subsubsection{Stochastic models}

The idea is to find stochastic models of turbulence that mimic the behaviour of Navier-Stokes equations at high Reynolds numbers, but which would be easier to solve numerically, and perhaps even analytically. These models could then be used to study some properties of turbulent flows, such as energy cascade, probability distribution functions, intermittency and departure from Kolmogorov's scaling.

The first attempt was done in 1974 by Desjanski and Novikov [46] who devised a so called shell model where the Navier-Stokes equations were represented on a discrete set of wavenumbers in Fourier space, each Fourier shell corresponding to one octave. The coupling between different octaves was supposed to be local in Fourier space and energy was transferred only from large to small scales. Such shell models, sometimes also called cascade models, are still popular because with them it is easy to obtain very large inertial range, up to Reynolds numbers $10^{10}$, at a limited computational cost. The number of degrees of freedom needed to compute threedimensional Navier-Stokes equations by standard direct simulations scale as $R e^{9 / 4}$, whereas they scale as $R e$ for shell models. The weak point of shell models is that the vectorial structure of Navier-Stokes equations is lost, the incompressibility condition is not satisfied and they do not give accurate information on the spatial structure of the flow. 
In 1981 Zimin [186], [79], [187] proposed another model, called the hierarchical model, defined in both space and scale. He projected the threedimensional Navier-Stokes equations onto Littlewood-Paley basis and discretized them by octaves, considering a limited number of vortices for each octave, few in the large scales and more in the small scales in accordance to the uncertainty principle. He then assumed that each vortex is advected by the velocity field of the larger vortices, which lead him to propose a set of semi-Lagrangian wavelets to compute the flow evolution. This impressive work fore-shadowed the wavelet decomposition, and has since been developed by Frick [78], [77], [7]. Hierarchical models are more physical than shell models because they also take into account the vortex motions, but they are still not very realistic from a physical standpoint because they neglect the vortex deformation which is responsible for energy transfers and subsequent dissipation. Recently Eyink, in an unpublished paper [62], has criticized this approach in showing that semi-Lagrangian wavelets do not remove the effect of large-scale convection to the energy transfers and therefore do not guarantee their locality (in wavenumber space). This is again due to Heisenberg's uncertainty principle and is related to the fact that it is impossible to compare transfers between wavenumbers and transfers between wavelets, this point has already been discussed in section 1.4.

Ideas on turbulence evolve at a very slow pace. As example of this, let us quote what Liepmann wrote in the proceedings of the turbulence conference held in Marseille in 1961 [124]: 'The success of the spectral representation of turbulent fields is due, after all, not to the belief in the existence of definite waves but to the possibility of representing quite general functions as Fourier integrals. In the application to stochastic problems the usefulness of the Fourier representation stems essentially from their translational invariance. Consequently, really successful models for representing turbulent shear flows will require far broader invariance considerations. It is clear that the essence of turbulent motion is vortex interaction. In the particular case of homogeneous isotropic turbulence this fact is largely masked, since the vorticity fluctuations appear as simple derivatives of the velocity fluctuations. In general this is not the case, and a Fourier representation is probably not the ultimate answer. The proposed detailed models of an eddy structure represent, I believe, a groping for an eventual representation of a stochastic rotational field, but none of the models proposed so far has proven useful except in the description of a single process'. These remarks, written 33 years ago, are still very pertinent and define the direction we should take for future research in turbulence.

Nowadays, using continuous wavelets we can construct more elaborate 
stochastic processes. As Liepmann has perceived we should be able to synthesize stochastic rotational fields, built from a set of randomly translated, rotated and dilated elementary vortices, which should have the same nonGaussian statistics as those observed for two and three dimensional turbulent flows. Recently Elliott and Majda [58], [59] have used wavelets to build a Gaussian, stationary and self-similar stochastic process for synthesizing turbulent velocity fields satisfying Taylor's hypothesis and displaying Kolmogorov's energy spectrum. Using these synthetic velocity fields they recover Richardson's law for scalar pair dispersion [60]. It is well-known that the Gaussian hypothesis is of somewhat limited validity in turbulence, but their method may be useful to model the background flow, which, contrary to coherent structures, does present Gaussian statistics.

\subsection{Turbulence computation}

\subsubsection{Direct Numerical Simulations}

The numerical simulation of turbulent flows, based on the direct integration of the Navier-Stokes equations at high Reynolds number without a sub-grid turbulence model, requires a very large number of degrees of freedom. This number increases like $R e$ in two dimensions and like $R e^{9 / 4}$ in three dimensions. Among the numerous Eulerian and Lagrangian numerical schemes, one may identify two different points of view: spectral and physical.

The first long-time simulations of two-dimensional turbulent flows [13], [127], [21] based on spectral methods, i.e. Fourier decomposition, had a resolution of $512^{2}$. More recently, resolutions of $4096^{2}$ have been calculated [41], but even these high-resolution simulations cannot attain realistic Reynolds numbers. On the other hand the physical point of view resulted in the development of Lagrangian methods ([1], e.g. vortex methods [118] or contour dynamics methods [112]) which follow the motion of each vortex, but which are imprecise concerning the background flow between the vortices. Finiteelement, -difference or -volume methods allow mesh refinement in regions of the flow where small structures appear, for instance in the boundary layer of an obstacle; unfortunately automatic adaptive refinements requires post-processing to follow these small structures.

Wavelet bases, in the context of the numerical simulation of PDEs (partial differential equations), appear to be a good compromise between spectral methods (precise, but expensive), contour dynamics (which automatically follow coherent structures, but not the background flow) and finite element or finite difference methods (local in space, of low order and therefore not precise). Wavelet numerical methods have already been used to solve Burg- 
ers' equation in one [8], [92] and two dimensions [24], Stokes' equation in two dimensions [174], the Kuramoto-Sivashinsky equation [142], BenjaminDavis-Ono-Burgers' equation [76], the heat equation in two dimensions [39], some reaction-diffusion equations in one and two dimensions [83] [28] [27], the non-linear Schrödinger equation [88], Euler's equation [155] and NavierStokes' equation in two dimensions [40], [85].

\subsubsection{Wavelet based numerical schemes}

The localization of wavelet bases, both in space and scale, leads to an effective nonlinear compression of the solution as well as of the operators involved in equations (1.1). Such a sparse representation is obtained by performing nonlinear thresholding of the wavelet coefficients of the solution and of the operator, i.e. those coefficients with absolute value below a given threshold are set to zero. This thresholding can be justified by theoretical results [47] and verified by numerical experiments.

The sparsity of the wavelet expansion of a given function is linked to its local smoothness: where the function is regular, the corresponding wavelet coefficients decrease with scale. This fact is related to the characterization of point-wise Hölder spaces [96], [93] (see subsection 1.3). Recall that for the Fourier decomposition, the decay of the coefficients depends on the global regularity of the function [188]. Another important property of wavelets is the nonlinear approximation of functions: the approximation error between a function and its wavelet series taken as the $N$ largest coefficients (in a given norm) can be estimated, in some Lebesgue space, by a (negative) power of $N$ which depends on the smoothness, or non-smoothness, of this function. This result follows from the characterization of Sobolev and Besov spaces by mean of wavelet coefficients [134], [47], [52]. Note that the nonlinear wavelet approximation of a given function is associated with a grid in physical space which is refined where there are singularities of this function.

A comparison of Fourier versus wavelet and wavelet packet nonlinear compression for a numerical vorticity field is shown in figure 1.7. We observe that the wavelet packet compression is the most efficient, both in terms of the minimal number of coefficients used and the quality of the approximation.

Another important consequence of the simultaneous localization in space and scale of wavelet bases is that many pseudo-differential operators and their inverse have a sparse representation, i.e. are almost diagonal or have a typical finger structure, depending on the employed form, i.e. non-standard or standard form [23]. This is the case for the gradient operators and the 
Fig. 1.7. Nonlinear compression of a vorticity field. In each case the strong coefficients (containing $95 \%$ of the total enstrophy) are displayed on the left, and the weak coefficients (containing $5 \%$ of the total enstrophy) are displayed on the right. (a) Uncompressed vorticity field computed with a resolution of $512^{2}$. (b) Compression in a Fourier basis (813 strong coefficients). (c) Compression in a wavelet basis (338 strong coefficients). (d) Compression in a wavelet packet basis (156 strong coefficients). 
Fig. 1.8. Discretization matrix of the heat operator $\left(1-10^{-4} \Delta\right)^{-1}$. The gray code is a logarithmic scale from white to black, the significant values being black. (a) In finite differences of fourth order. (b) In a wavelet basis with the same precision.

heat kernel. For a theoretical justification in the general context of CalderonZygmund operators we refer the reader to [134].

As an example, the discretized heat kernel (on a $1024^{2}$ grid) is projected onto a wavelet basis (figure 1.8b) and we observe that only $9.5 \%$ of the coefficients are greater than $10^{-8}$, absolute value to be compared to the largest eigenvalue which is order 1 , instead of $21 \%$ for a finite difference projection (figure 1.8a).

These two fundamental properties (compression of the solution and of the operator) allow us to define adaptive wavelet-based numerical schemes for solving PDE's. By neglecting small coefficients in the solution and/or in the operator's wavelet representation, each step of the algorithm is based on approximate but fast matrix-vector products computed in wavelet space.

Note that the schemes based on scaling functions (often deliberately confused with wavelets) [90], [109], [76] instead of wavelet functions are no more efficient than classical finite element methods on a regular grid! Theoretical 
error and stability estimates for some particular wavelet schemes may also be derived [23], [44], [25]. A scaling function scheme for solving the Euler equations has already been developed by Qian and Weiss [155].

\subsubsection{Solving Navier-Stokes equations in wavelet bases}

Before presenting wavelet-based numerical schemes to solve the NavierStokes equations, we should mention a very interesting direction which consists of simplifying the Navier-Stokes equations by re-writing them in an appropriate wavelet basis. Jacques Lewalle has shown that some continuous wavelets, namely the Hermitian wavelets (derivatives of the Gaussian), simplify the resolution of the linear term and allow a simpler convolution formula for the nonlinear term [120], [121]. He has found that the first derivative of the Gaussian gives a Hamiltonian form of the diffusion equation, where dissipation is replaced by spectral transport, namely Hermitian wavelets are propagators for the diffusion equation [122].

The first wavelet adaptive schemes for the Navier-Stokes equations, have been derived by Charton \& Perrier [38] and Fröhlich \& Schneider [85]. Different approaches can be used to solve the two-dimensional Navier-Stokes equations. We will focus here on the two recently developed wavelet schemes for solving Navier-Stokes equations: the algebraic wavelet method of Charton \& Perrier [40] and the Petrov-Galerkin scheme of Fröhlich \& Schneider $[85,84]$. Both methods are based on the discrete wavelet transform and use take advantage of the nonlinear compression of the operators and the solution.

Apart from the above Eulerian schemes another possible approach would be to develop Lagrangian-type wavelet methods, based on the continuous wavelet transform. The travelling wavelet method in which wavelets behave like particles evolving in phase-space coordinates has been proposed in 1990 by Basdevant, Holschneider and Perrier [12]. The travelling wavelet method looks for an approximate solution of the above equation (1.50) which is a finite sum of wavelets evolving in phase-space:

$$
\omega(x, t) \approx \sum_{i=1}^{N} c_{i}(t) \psi\left(\frac{x-b_{i}(t)}{a_{i}(t)}\right), a_{i}>0,
$$

where $\psi$ is the base-wavelet and $c_{i}, a_{i}, b_{i}$, are respectively the time dependent amplitude, scale and position parameters.

This method works well for linear equations, such as the convectiondiffusion equation, and also for the Korteweg-de-Vries equation. It has also very recently been applied to the study of the formation of galaxies [19]. 
However, in the nonlinear case the method encounters technical difficulties which have not yet been completely overcome. These difficulties arise when two wavelets approach each other in phase-space which leads to a "phasespace atom collision".

Now let us consider the two-dimensional Navier-Stokes equations written in terms of vorticity and stream function, which are pseudo-scalars

$$
\left\{\begin{array}{l}
\frac{\partial \omega}{\partial t}+\boldsymbol{v} \cdot \nabla \omega=\nu \nabla^{2} \omega+f, \quad x \in[0,1]^{2}, t>0 \\
\nabla^{2} \Psi=\omega, \boldsymbol{v}=\left(\frac{\partial \Psi}{\partial y},-\frac{\partial \Psi}{\partial x}\right),
\end{array}\right.
$$

We complete the problem with periodic or Dirichlet or Neumann boundary conditions and a suitable initial condition.

By introducing a time step $\delta t$ and a classical semi-implicit time discretization, and setting $\omega^{n}(x) \approx \omega(x, n \delta t)$ to be the approximate solution at time $n \delta t$, equation (1.50) is replaced, for example (for notational ease we take here the simplest, but unstable, time scheme), by

$$
\left\{\begin{array}{l}
\left(1-\nu \delta t \nabla^{2}\right) \omega^{n+1}=\omega^{n}+\delta t\left(f^{n}-\mathbf{v}^{\mathbf{n}} \cdot \nabla \omega^{\mathbf{n}}\right) \\
\nabla^{2} \Psi^{n+1}=\omega^{n+1}, \boldsymbol{v}^{n+1}=\left(\partial_{y} \Psi^{n+1},-\partial_{x} \Psi^{n+1}\right)
\end{array}\right.
$$

The spatial discretization is then performed by approximating, at time $n \delta t$, $\omega^{n}$ by a function $\omega_{J}^{n}$ belonging to a finite dimensional subspace $V_{J}$ obtained from a multi-resolution analysis $\left(V_{j}\right)_{j \geq 0}$ of the space $L^{2}\left([0,1]^{2}\right)$.

At this point the algebraic method of Charton \& Perrier differs significantly from the Petrov-Galerkin scheme of Fröhlich \& Schneider. In [40] one starts with a finite difference scheme on a regular Cartesian grid. Wavelets are then used to speed up the solution procedure by compression of the discrete inverse operator and the actual solution during the time advancement. Furthermore, operator splitting by means of an ADI (Alternating-DirectionImplicit) technique is introduced. The two-dimensional wavelet basis employed relies on a tensor product of two one-dimensional multi-resolution analyses. The method of $[84,85]$ uses a two-dimensional multi-resolution analysis as the underlying wavelet basis. In this case the inverse operator is applied during the time advancement, using special test functions.

We will attempt to clarify the basis of these wavelet methods. In principle, the spatial approximation can be of collocation type, i.e. grid point values, or of Galerkin type, i.e. a projection onto a basis. The transformation between the single level representation of a function, i.e. at regular collocation points, and a multi-level wavelet Galerkin representation uses an orthogonal wavelet transform. However, problems arise with adaptive schemes because it is difficult to take advantage of the sparsity of the wavelet decomposition when going back and forth between grid point and wavelet representations. Let 
us be more precise, and consider the one-dimensional case. Suppose that $\operatorname{dim} V_{J}=2^{J}$. Then the function $\omega_{J}^{n}$ can be expanded onto the scaling function basis (single level representation) $\left(\varphi_{J, k}\right)_{k=0,2^{J}-1}$ of $V_{J}$

$$
\omega_{J}^{n}(x)=\sum_{k=0}^{2^{J}-1} c_{J, k}^{n} \varphi_{J, k}(x),
$$

or onto a wavelet basis $\left(\psi_{j, k}\right)_{0 \leq j \leq J, k=0,2^{j}-1}$ of $V_{J}$

$$
\omega_{J}^{n}(x)=\sum_{j=0}^{J} \sum_{k=0}^{2^{j}-1} d_{j, k}^{n} \psi_{j, k}(x)+c_{0,0}^{n} .
$$

The transition between both representations is done by the orthogonal wavelet transform (Mallat's algorithm).

In the collocation method, the function $\omega_{J}^{n}$ is naturally associated with a regular grid $\left(x_{k}=k 2^{-J}\right)_{k=0,2^{J}-1}$ of $[0,1]$ and its corresponding collocation values $\omega_{J}^{n}\left(x_{k}\right)$. Often, by using properties of scaling functions $\varphi_{J, k}$ one can identify

$$
\omega_{J}^{n}\left(x_{k}\right) \approx 2^{-J} c_{J, k}^{n} .
$$

The wavelet Galerkin method is based on the wavelet coefficients $d_{j, k}^{n}$, and in practice uses only the few (non-negligible) coefficients larger than a given threshold $\varepsilon:\left\{d_{j, k}^{n} ;\left|d_{j, k}^{n}\right|>\varepsilon\right\}$. Mallat's fast wavelet algorithm works well for regular grids, but is not efficient for irregular grids made up of irregularly spaced grid points $x_{k}$ corresponding to the "centers" of wavelets $\psi_{j, k}$, for which the coefficients of $\omega_{J}^{n}\left(x_{k}\right)$ satisfy $\left|d_{j, k}^{n}\right|>\varepsilon$.

To avoid this problem, one can introduce, when it exists, an interpolating function of $V_{J}$ [179] and adapt Mallat's fast wavelet algorithm [82, 84]. Another way to overcome this problem is to directly construct the interpolating scaling functions $\varphi_{J, k}$ and the corresponding interpolating wavelet basis $\psi_{j, k}$ [51] [24]. Finally, one can also construct an adaptive multi-resolution analysis [154], [3].

The algorithm (1.51) for solving the two-dimensional Navier-Stokes equations can now be split into four steps which we will discuss below: 1 . timestepping the heat equation, 2. solving a Poisson equation, 3. computing the non-linear term, 4. imposing the boundary conditions.

\subsubsection{The heat equation solution}

Let us consider the discretized heat equation

$$
\left(1-\nu \delta t \nabla^{2}\right) \omega^{n+1}=\omega^{n}+\delta t f^{n} .
$$


The biorthogonal approach introduced in [123], [111], [83] [84] consists of building a biorthogonal system from a classical wavelet basis $\psi_{j, k}$, first setting

$$
\theta_{j, k}=\left(1-\nu \delta t \nabla^{2}\right)^{-1} \psi_{j, k},
$$

with suitable hypotheses on $\psi$. Then a system $\tilde{\theta}_{j, k}$ biorthogonal to $\theta_{j, k}$ is constructed, and equation (1.55) is reduced to the change of bases

$$
\left\langle\omega^{n+1} \mid \psi_{j, k}\right\rangle=\left\langle\omega^{n} \mid \theta_{j, k}\right\rangle+\delta t\left\langle f^{n} \mid \theta_{j, k}\right\rangle,
$$

where the notation $\langle\mid\rangle$ means scalar product. The functions $\theta_{j, k}$ and $\tilde{\theta}_{j, k}$ are called vaguelettes and have localization properties similar to those of wavelets [134]. This approach avoids assembling and solving a linear system. For the collocation projection operator-adapted cardinal functions [84] have been constructed which allow the construction of efficient interpolatory quadrature formulas. The decomposition of the rhs of equation (1.57) can then be calculated using the fast adaptive vaguelette decomposition of [84] based on a hierarchical subtraction strategy. This approach has been used for one- and two-dimensional problems.

The Galerkin approach is to project (1.55) onto a classical, orthogonal or biorthogonal, wavelet basis $\left(\psi_{j, k}\right)$ of the space $V_{J}$. We can write

$$
\left(\left\langle\omega_{J}^{n+1} \mid \psi_{j, k}\right\rangle\right)_{j, k}=\mathrm{K}\left(\left\langle\omega_{J}^{n}+\delta t f \mid \psi_{j, k}\right\rangle\right)_{j, k}
$$

where

$$
\mathrm{K}_{(j, k),\left(j^{\prime}, k^{\prime}\right)}=\left\langle\left(1-\nu \delta t \nabla^{2}\right)^{-1} \psi_{j, k} \mid \psi_{j^{\prime}, k^{\prime}}\right\rangle
$$

is the heat kernel, which is almost diagonal, as explained in section 1.6.2, figure 1.8(b). This step is based on approximated, but fast, matrix-vector products. An easy way to reduce the previous two-dimensional system to several one-dimensional systems is to use a tensor wavelet basis $\left(\psi_{j, k}(x) . \psi_{j^{\prime}, k^{\prime}}(y)\right)$ and to split the two-dimensional heat kernel into two one-dimensional operators

$$
\left(1-\nu \delta t \nabla^{2}\right)^{-1} \approx\left(1-\nu \delta t \frac{\partial^{2}}{\partial x^{2}}\right)^{-1}\left(1-\nu \delta t \frac{\partial^{2}}{\partial y^{2}}\right)^{-1}
$$

as in ADI method. Such a method is applied in [39], [40].

\subsubsection{The Poisson equation}

The solution to the Poisson equation

$$
\nabla^{2} \Psi^{n+1}=\omega^{n+1}
$$


can be obtained using a pseudo-transient technique, i.e. calculating the steady state solution of the heat equation, which, as in ADI methods, is reached in only a few iterations by considering iterated powers $\mathrm{K}^{n}$ of the heat kernel K (1.59) which become sparser with $n$ [39].

An alternative approach, proposed by Jaffard [96], is to consider the wellconditioned system

$$
\text { P A P P }{ }^{-1}\left(\left\langle\Psi_{J}^{n+1} \mid \psi_{j, k}\right\rangle\right)_{(j, k)}=\mathrm{P}\left(\left\langle\omega_{J}^{n+1} \mid \psi_{j, k}\right\rangle\right)_{(j, k)}
$$

where $\mathrm{A}$ is the Galerkin matrix of the Laplacian in a wavelet basis: $\mathrm{A}_{(j, k),\left(j^{\prime}, k^{\prime}\right)}=$ $\left\langle\nabla^{2} \psi_{j, k} \mid \psi_{j^{\prime}, k^{\prime}}\right\rangle$ and $\mathrm{P}$ is the diagonal pre-conditioning matrix: $\mathrm{P}_{(j, k),\left(j^{\prime}, k^{\prime}\right)}=$ $2^{-j} \delta_{j, j^{\prime}} \delta_{k, k^{\prime}}$, in one dimension (in two dimension this should be modified according to the chosen $2 \mathrm{D}$ wavelet basis). Jaffard proved that the condition number of PAP does not depend on the order of the system. Then the solution of (1.62) can be reached in a few iterations by a classical conjugate gradient method.

The biorthogonal approach is also possible using operator-adapted biorthogonal vaguelettes for homogeneous operators, i.e. $\theta_{j, k}=\left(\nabla^{2}\right)^{-1} \psi_{j, k}$ and $\tilde{\theta}_{j, k}=\nabla^{2} \psi_{j, k}$. The solution of the Poisson equation then also reduces to a change of basis, analogously to case of the heat equation.

\subsubsection{The nonlinear term}

The nonlinear term $\boldsymbol{v}^{n} \cdot \nabla \omega^{n}$ can be computed either by a collocation or by a Galerkin method. The collocation (also called pseudo-spectral) method can be sketched as follows: starting from the wavelet coefficients of $\boldsymbol{v}^{n}$ and $\omega^{n}$, compute the wavelet coefficients of $\nabla \omega^{n}$. Then, through an inverse wavelet transform, obtain the grid point values of $\boldsymbol{v}^{n}$ and $\nabla \omega^{n}$ on the associated grid, as above. Then the products are calculated at each grid point, and finally the wavelet coefficients of the nonlinear term are obtained by a direct wavelet transform. This collocation method requires a fast wavelet transform between grid points and sparse coefficients sets. This problem was mentioned in the previous section 1.6.3. Fröhlich and Schneider [84, 82] have developed a wavelet transform for lacunary bases which enables the adaptive evaluation of terms of the form $f(\omega)$ without derivatives. This method has been applied for the full adaptive discretization of reaction-diffusion problems [27]. Recently, this approach has been extended to convective terms [86], i.e. nonlinear terms of the form $f(\omega, \nabla \omega)$. Consequently the implementation of this algorithm will enable the adaptive evaluation of the convective term.

On the other hand, a Galerkin method works only in the wavelet coefficient space, avoiding transforms between physical and wavelet space [22]. 
The nonlinear term is then written as a convolution of the wavelet coefficients of $\boldsymbol{v}^{n}$ and $\nabla \omega^{n}$; these convolutions involve triple wavelet connection coefficients of the form $\left\langle\psi_{j_{1}, k_{1}} \psi_{j_{2}, k_{2}}^{\prime} \mid \psi_{j_{3}, k_{3}}\right\rangle$. A priori the complexity of such a calculation is very large, but the method can be competitive for two reasons. First, since the wavelets are localized both in space and scale, connection coefficients vanish when two of the three wavelets are separated either in scale or space. Hence, only a small number of terms in the convolution are significant. Secondly, the method can, more easily than collocation, handle adaptive description of the fields, i.e. the convolution can be restricted to the significant components of the flow [150].

Let us mention that at the moment in both methods $[40,85]$ the nonlinear term is computed by a collocation method on a regular grid. This aspect will be improved in the near future.

\subsubsection{The boundary conditions}

Boundary conditions are in general included in the definition of the spaces $\left(V_{j}\right)_{j \in Z}$ when constructing the multi-resolution analysis. The simplest and most popular (due to the development of Fourier spectral methods) are periodic boundary conditions for which periodic wavelets, in one or several dimensions, can be easily constructed [148]. For Dirichlet, or Neumann boundary conditions, compactly supported bases have recently been constructed in one dimension [42], [139], [140], and these bases are also associated to fast orthogonal wavelet transforms, like for the periodic case. They can easily be included in some of the previous algorithms, since the extension to cubic domains in several dimensions is trivial using tensor products of wavelets (in practice all two-dimensional orthogonal wavelet bases are tensor products, which raises the problem of the lack of isotropy).

One should also mention the existence of divergence-free wavelet bases [116], [115], which can be used for the velocity-pressure formulation of NavierStokes equation (1.1) and automatically take into account the incompressibility condition. This approach has been used for the linearized NavierStokes equations, the Stokes problem [174].

\subsubsection{Numerical results}

To illustrate the previously described adaptive wavelet methods we present some numerical results for two different cases, i.e. a strong nonlinear interaction of three vortices and a decaying turbulent flow. For comparison a classical pseudo-spectral method serves as a reference. Furthermore in order to study statistically stationary turbulent flows we discuss results computed 
with a recently developed wavelet based forcing method [74]. In all computations presented below the method of [84] using cubic spline wavelets of Battle-Lemarié type have been used.

\subsubsection{Three vortex interaction}

As a prototype for vortex merging we consider the strong nonlinear interaction of three Gaussian vortices [166]. For details on the numerical simulation we refer the reader to [166]. The initial condition is given by the superposition of two positive and one negative Gaussian vortices, $\omega(x, y)=$ $\sum_{i=1}^{3} A_{i} \exp \left(-\left(\left(x-x_{i}\right)^{2}+\left(y-y_{i}\right)^{2}\right) / S_{i}^{2}\right)$ with amplitudes $A_{1}=A_{2}=$ $-2 A_{3}=\pi$ and variance $S_{i}=1 / \pi$. The maximum resolution of the computation corresponds to a finest scale $J=8$ which is equivalent to $256^{2}$ possible degrees of freedom. As threshold for the adaptive method [84] we used $\varepsilon=10^{-6}$, i.e. only wavelet coefficients with absolute value larger than $\varepsilon$ have been computed.

In figure 1.9 we show the vorticity field for the reference pseudo-spectral method and the adaptive wavelet method with the corresponding computed wavelet coefficients (dark entries) at for instants, $t=10,20,30$ and 40. We observe that during the interaction high frequency components are produced, which is directly reflected in the active wavelet coefficients. For the initial condition only about $3 \%$ of the wavelet coefficients, the strongest ones. At later times the number of active coefficients increases to $20 \%$, i.e. we still have a compression of a factor 5 . The comparison of the vorticity fields with the pseudo-spectral method, see figure 1.9, shows no significant difference. If we look at the energy spectra at $t=40$ we can observe quantitatively that all relevant scales, in particular the small ones, are well resolved. However, as the fine resolution is only required locally, the number of degrees of freedom has in comparison to the pseudo-spectral method been reduced by a factor 5 .

Let us mention that at the moment both existing adaptive wavelet methods $[40,84,85]$ are not yet more efficient in terms of computing time than a classical, well-optimized, pseudo-spectral method. In principle the adaptive wavelet methods have a computational complexity of order $N_{a d}$, where $N_{a d}$ denotes the number of the degrees of freedom adapted to the solution. In comparison the pseudo-spectral methods are of $O\left(N_{\text {reglog }} \log _{2} N_{\text {reg }}\right)$ complexity, where $N_{\text {reg }}$ denotes the number of degrees of freedom on the regular grid. The actual numerical cost depends directly on the constant multiplying the order term. At the moment this factor is rather high for the adaptive wavelet methods. For simulations at moderate resolutions, such 
Fig. 1.9. Simulation of the merging of three vortices at times $t=10,20,30,40$. (a) Vorticity field, reference pseudo-spectral method. (b) Vorticity field, adaptive wavelet method. (c) Wavelet coefficients used in the adaptive wavelet method. (d) Comparison of Fourier energy spectra for the pseudo-spectral and adaptive wavelet methods (note that the two curves are essentially identical). 
as $128^{2}$ or $256^{2}$, the adaptive wavelet methods cannot yet outperform the classical spectral methods.

\subsubsection{Decaying turbulence}

For the computation of decaying turbulence one typically uses a statistical initial condition, generated by means of Gaussian random numbers and imposing a given energy spectrum. Here we used a broad band spectrum of the form $E(k)=c k^{2} /\left(k_{0}{ }^{6}+k^{6}\right) \exp \left(-k^{2} / k_{\nu}{ }^{2}\right)$ with $k_{0}=10$ and $k_{\nu}=80$. The constant $c$ has been chosen such that the total kinetic energy was equal to $1 / 2$. The maximal resolution was $256^{2}$ numbers of degrees of freedom, with $\nu=10^{-3}$. Using a classical pseudo-spectral method we calculated the solution up to $t=4$ corresponding to 12 initial eddy turn-over times. The resulting vorticity field, exhibiting coherent structures and a smooth spectrum with an inertial range, was then taken as initial condition for the adaptive wavelet calculation and therefore we assigned the time $t=0$. The threshold for the wavelet coefficients was $\varepsilon=510^{-5}$. In figure 1.10 we give an example of the vorticity field at $t=2$ for the pseudo-spectral method and the adaptive wavelet method with the corresponding wavelet coefficients which have been computed. As observed in the case of the three vortices the wavelet solution does not exhibit a visible difference with respect to the spectral method. However, out of the total $256^{2}$ wavelet coefficients, only about $20 \%$ have been used during the calculation of the solution. The energy spectrum also does not deviate significantly from the reference, thus we may conclude that all scales are well-resolved with only $1 / 5$ th of the possible degrees of freedom. We should mention that the resolution of the present calculations with $256^{2}$ is fairly small. Since for higher resolutions, larger Reynolds number flows can be computed the compression rate of the wavelet representation will increase due to the greater intermittency of the flow. Therefore the impact of adaptive wavelet methods will become particularly attractive for high Reynolds number flows.

\subsubsection{Wavelet-forced turbulence}

The numerical simulation of turbulent flows has been performed considering two different regimes: either the decaying regime, where the flow is excited initially and its evolution is computed without any forcing, or the forced regime, where the flow is excited in such a way that it reaches a statistically steady state for which the dissipation must be compensated by the forcing. The advantage of the decaying regime is that it depends only on the flow's intrinsic nonlinear dynamics, with the hope of thus observing a universal behaviour. The problem with this method is that it never reaches 
Fig. 1.10. (a) Vorticity field of the decaying turbulence simulation at time $t=2$ for the reference pseudo-spectral method. (b) The vorticity field at $t=2$ for the adaptive wavelet method. (c) The Fourier energy and enstrophy spectra for the two methods. (d) The corresponding wavelet coefficients used by the adaptive wavelet method. 
a statistically steady state because energy or enstrophy tends to decay in time. The advantage of the forced regime is that the turbulent flow reaches a statistically steady state, but this state depends on the kind of forcing performed [13], which precludes a universal turbulent behaviour.

Classically, two forcing schemes are used [13] which both operate in Fourier space. Either a negative dissipation within a given wavenumber band, with a complex amplification coefficient which depends on the wavenumber, or a white or coloured noise in time with a prescribed isotropic spectral distribution, strongly peaked in the vicinity of a given wavenumber, with random phases. For both schemes the choice of the wavenumber band represents that part of the energy spectrum where the baroclinic instability has a significant growth rate. Neither of the two schemes is a satisfactory model because they inject energy and enstrophy locally in Fourier space and therefore non-locally in physical space. This forcing mechanism is neither intrinsically related to the flow's chaotic dynamics, nor simulates the production of enstrophy in shear layers, which is local in physical space and therefore broad-band in Fourier space. Another drawback of such a forcing is that the scale of the coherent vortices produced by the nonlinear dynamics of the flow is imposed by the scale at which the forcing is done. We have designed [74] a forcing scheme able to excite vortices locally in physical space and as smoothly as possible in order to avoid creating any unphysical discontinuities in the vorticity field.

Here we apply a new nonlinear wavelet-based forcing approach, which is triggered directly by the intrinsic nonlinear dynamics of the flow. It has been shown that vortices produced in two-dimensional turbulent flows correspond to the strongest wavelet coefficients of the vorticity field while the remaining weaker coefficients correspond to the residual background flow $[73,64,65,71]$. Furthermore we include an artificial dissipative term $\lambda \Psi$, a so-called Rayleigh friction [13], to provide an energy sink at large scales. This is necessary because the energy injected by external forcing tends to accumulate in the large scales due to the inverse energy cascade characteristic of two-dimensional turbulent flows and should therefore be dissipated there in order to reach a statistically stationary regime. Therefore the forcing scheme we propose injects enstrophy only into the strongest wavelet coefficients, hence in an inhomogeneous way, in order to excite the vortices without affecting the background flow. This procedure does not interfere with the emergence of vortices and does not impose them a scale on them, contrarily to the Fourier forcing. The distribution and size of the vortices depend only on the intrinsic nonlinear dynamics of the flow.

For the numerical results presented here both energy and enstrophy are 
kept steady during more than 60 eddy turn over times. Figure 1.11(a) displays the vorticity field in a stationary regime at $t=0,6$ and 12 , namely neither the energy spectrum (figure 1.11c) nor the PDF of vorticity (figure 1.11d) change significantly in time. The vortices present in the initial condition become more circular and well isolated during the flow evolution because they are better able to withstand the mutual strain due to the additional enstrophy injected into them. We observe that the slopes of the spectra (see figure 1.11c) are much steeper (close to $k^{-6}$ ) than the $k^{-3}$ law predicted by the statistical theory of homogeneous turbulence. This discrepancy, as observed for other types of forcing [13], confirms the fact that the spectral behaviour of two-dimensional turbulent flows is not universal, but instead depends on the forcing. In figure 1.11(b) we observe that the spatial support of the active wavelet coefficients decreases with the scale, which reveals a strong intermittency of the flow. Consequently the vorticity field is efficiently compressed in a wavelet basis, because only about $20 \%$ of the $128^{2}$ coefficients are needed to represent the flow dynamics. We also show that the PDF of vorticity (figure 1.11b) is Gaussian for the weak values, corresponding to the background flow, and presents non-Gaussian tails for the strong values, corresponding to the vortices.

In the work presented here, we only excite the vortices produced by the flow's nonlinear dynamics. We can also use the same wavelet forcing to create new vortices by injecting enstrophy locally in the regions of the background flow where the strain becomes weaker than the vorticity, in order to simulate the formation of new vortices by instabilities, such as KelvinHelmholtz instability.

\subsection{Conclusion}

The main factor limiting our understanding of turbulent flows is that we have not yet identified the structures responsible for its chaotic and therefore unpredictable behaviour. Based on laboratory and numerical experiments, we think that vortices (or coherent structures) are these elementary objects, from which we may be able to construct a new statistical mechanics and define equations appropriate for computing fully developed turbulent flows.

The quasi-singular vortices encountered in turbulent flows are, by their nature, very rare. In fact, the Cafarelli, Kohn and Nirenberg theorem shows that singular structures, if they exist, must be of Hausdorff measure one in space-time. The present statistical diagnostics are low order and thus insensitive to rare events, but the effect of coherent structures appears only in the higher order statistics. An example of this is the fact that the low 
Fig. 1.11. Temporal evolution of the wavelet-forced turbulence simulation. (a) The vorticity field at $t=0,60,120$. (b) The wavelet coefficients used at $t=0,60,120$. (c) The Fourier energy and enstrophy spectra at $t=0,60,120$. (d) The PDF of vorticity at $t=0,60,120$. 
order structure functions follow Kolmogorov's 1941 law (which assumes a homogeneous structureless and non-intermittent flow), while the higher order structure functions depart strongly from this law (because turbulent flows are actually highly intermittent). To efficiently analyse the coherent structures of turbulence one requires either a high order statistical method or some conditional averaging.

Using a wavelet representation instead of a Fourier representation minimizes the restrictions on the basis functions enlarging them to Sobolev, Hölder and Besov spaces. Moreover, the Fourier basis used by the present statistical theory of turbulence is not the appropriate functional representation space for analysing the physical structure of a flow because it averages over space and thus loses all spatial information. Furthermore, the Fourier energy spectrum is sensitive to only the strongest isolated singularity in the flow, and even then can give no information about the form or location of this singularity. In short, Fourier space analysis is unable to disentangle coherent structures from the rest of the flow.

The complementary simultaneous space and scale information provided by the wavelet representation makes it an appropriate tool for identifying and analysing coherent structures in turbulent flows. The wavelet transform can be used to segment the vorticity field into coherent and incoherent components as the first stage in a conditional sampling algorithm. Such a segmentation method respects Galilean invariance because it is performed on the vorticity field and not on the velocity field, which loses Galilean invariance. A local wavelet analysis can also give the strength and form of any quasi-singular isolated structures, which correspond to the coherent components, and separate them from the background flow, which corresponds to the incoherent components.

Different wavelet techniques must be used depending on whether the flow contains oscillating (e.g. spiral) or non-oscillating (e.g. cusp) type singularities, and whether it contains isolated (e.g. a single cusp or spiral) or dense (e.g. fractal) distributions of singularities. For example, the current wavelet-based methods for determining the singularity spectrum of a multifractal work only if the signal does not contain oscillating singularities. Turbulence may contain both types of singularities in either dense or isolated distributions. It is therefore important to determine from the beginning whether a given turbulence signal contains oscillating singularities and how these singularities are distributed. This classification is possible using a wavelet-based diagnostic.

In section 1.3 we reviewed the wavelet-based methods for detecting and analysing the singular structure of a signal. We saw that these methods 
are useful, not only because they provide new information which cannot be obtained using other methods, but also because they formally unify a wide range of previously disparate approaches. For instance the wavelet-based method of calculating the structure functions unifies their analysis with the calculation of energy spectra and the strength of local singularities. Furthermore, wavelets play the role of 'generalized boxes' in a new form of the standard box-counting algorithm used to estimate fractal dimensions. This algorithm brings out the intimate relationship between structure functions and multifractals. The application of these methods to turbulence is still in its first stages, although they have already produced interesting and stimulating new results.

In section 1.4 we showed that wavelet analysis has been an essential tool for identifying coherent structures as phase-space regions correlated in both space and scale, and for studying their scaling properties. Wavelet analysis has helped to relate the intermittency of turbulent flows to the presence of organized coherent structures, and explained why the predictions of the statistical theory of turbulence are not verified for high-order statistics. The wavelet representation may also be used to compute the transfers of energy and of enstrophy between coherent and incoherent components of turbulent flows.

In section 1.5 we reviewed several applications of wavelets and wavelet packets to turbulence modelling. In particular, we showed that the wavelet packet representation, associated with a maximum entropy statistical method and a nonlinear filtering procedure, extracts the coherent structures in a computationally efficient way. Turbulent motions are non-separable in the Fourier representation, while a wavelet representation may be able to provide such separability. We have reasons to expect a gap in wavelet coordinates between organized structures to be explicitly computed and random background flow to be modelled by an appropriate stochastic process. This decomposition may be the basis of a new way of numerically simulating turbulent flows and possibly other kind of intermittent behaviour having similar statistics.

In section 1.6 we summarized the progresses that has been made in actually computing partial differential equations in wavelet space. Numerous promising experiments have been carried out using wavelets on Burgers' equation in one or two dimensions, heat equation or Stokes equation in two dimensions and Navier-Stokes equations in two dimensions. All these experiments have shown that wavelet approaches are valid and sometimes superior to existing numerical methods.

In conclusion, we think that the wavelet functional representation may be 
the proper tool for building a statistical mechanics of turbulence based on the identification of elementary dynamical structures from the observational data we have. This theory will replace the present Fourier-space statistical theory of turbulence which is based on the symmetries of the Navier-Stokes equations. We are now convinced that the Navier-Stokes equations are not the practical model equations to compute large Reynolds number flows. Indeed in this limit, there is probably some symmetry breaking associated with the production of coherent structures out of the random background flow. This is precisely the difference between a statistical theory and statistical mechanics!

Turbulence research is a kind of tragi-comedy - tragic due to its military (atomic bomb, missiles, reentry vehicles) applications - and comic because at each generation we seem fated to rediscover old ideas. For instance, our understanding of dissipation and turbulence modeling is the same as what Richardson was suggesting 66 years ago when he wrote 'Diffusion is a compensation for neglect of detail. By an arbitrary choice we try to divide motions into two classes: (a) Those which we treat in detail. (b) Those which we smooth away by some process of averaging' [160], and the program we develop corresponds to the prescription for turbulence research proposed 48 years ago by Dryden when he wrote: 'It is necessary to separate the random processes from the non-random element' [54].

Wavelets, as a new mathematical tool, will certainly bring new insights to evaluate current methods and we hope that they will lead to a better understanding of turbulent flows. But, knowing the past difficulties encountered in this field, we should not be overly optimistic, nor should we over-sell wavelets. As Robert Sadourny likes to say ironically: 'Wavelets? You mean this new approach which will waste another 20 years of turbulence research!'.

\section{Acknowledgments}

This work has been supported by the NATO 'Collaborative Research' on 'Wavelet Methods in Computational Turbulence' (contract CRG-930456), by the Training and Mobility of Researchers programme of the EC (contract ERBFMBICT950365) and by the French-German PROCOPE programme (contract 95137). These grants are gratefully acknowledged. We thank Echeyde Cubillo, Eric Goirand and Thierry Philipovitch who computed some of the results presented here. We also thank Claude Bardos, Hans van den Berg, Stéphane Jaffard, Abdelhaq Hamza, Jacques Lewalle, Stéphane Mallat and Igor Mezić for reading this chapter and making useful comments. 


\section{Bibliography}

[1] Abgrall, R. and Basdevant, C. (1987). Un schéma numérique semi-Lagrangien pour la turbulence bidimensionelle, C. R. Acad. Sci. Paris, 305, série I, 315-318.

[2] Abry, P., Fauve, S., Flandrin, P. and Laroche, C. (1994). Analysis of pressure fluctuations in swirling turbulent flows, J. Physique II, 4, $725-733$.

[3] Amellaoui, A. (1996). Ondelettes splines à support compact sur une grille irrégulière et approximations numériques stables. Thèse de doctorat de l'université Paris 11.

[4] Anselmet, F., Gagne, Y., Hopfinger, E. J., and Antonia, R. A. (1984). High-order velocity structure function in turbulent shear flows, J. Fluid Mech., 140, 63-89.

[5] Arnéodo, A., Bacry, E. and Muzy, J. F. (1995). The thermodynamics of fractals revisited with wavelets, Physica A., 213, 232-275.

[6] Arnéodo, A., Bacry, E. and Muzy, J. F. (1995). Oscillating singularities in locally self-similar functions, Phys. Rev. Lett., 74(24), 4823-4826.

[7] Aurell, E., Frick, P., and Shaidurov, V. (1994). Hierarchical tree-model of 2D-turbulence, Physica D, 72, 95-109.

[8] Bacry, E., Mallat, S., and Papanicolaou, G. (1992). A wavelet based space-time adaptive numerical method for partial differential equations, Math. Mod. Num. Anal., 26, 793.

[9] Balian, R. (1981). Un principe d'incertitude fort en théorie du signal ou en mécanique quantique, C. R. Acad. Sci. Paris, 292, II, 1357-1361.

[10] Bardos, C., Golse, F., and Levermore, D. (1991). Fluid dynamical limit of kinetic equations: 1. formal derivations, J. Stat. Phys., 63, 323-344.

[11] Bardos, C., Golse, F., and Levermore, D. (1993). Fluid dynamical limit of kinetic equations: 2. convergence proofs for the Boltzmann equation, Comm. Pure Appl. Math., 46(5), 667-754.

[12] Basdevant, C., Holschneider, M., and Perrier, V. (1990). Méthode des ondelettes mobiles, C. R. Acad. Sci. Paris, série I, 310, 647-652.

[13] Basdevant, C., Legras, B., Sadourny, R., and Béland, M. (1981). A study of barotropic model flows: intermittency waves and predictability, J. Atmos. Sci., 38, 2305-2326.

[14] Batchelor, G. K. (1969). Computation of the Energy Spectrum in Homogeneous Two-dimensional Turbulence, Phys. Fluid, suppl. II, 12, 233-239.

[15] Batchelor, G.K. (1953). Homogeneous turbulence, (Cambridge University Press).

[16] Batchelor, G. K., and Townsend, A. A. (1949). The Nature of turbulent motion at large wave-numbers, Proc. Roy. Soc. Lond. A, 199, 238-255.

[17] Battimelli, G. (1984). The mathematician and the engineer: the statistical theories of turbulence in the 20's, Riv. Stor. Sci., 1(1), 73-94.

[18] Battle, G., and Federbush, P. (1993). Divergence-free vector wavelets, Michigan Math. J., 40, 181-195.

[19] Benhamidouche, N. (1995). Ondelettes mobiles et l'instabilité gravitationnelle, Thèse de Doctorat de l'Université d'Aix-Marseille II.

[20] Benzi, R., Ciliberto, S., Baudet, C., Ruiz-Chavarria, G. and Tripiccione, R. (1993). 'Extended self-similarity in the dissipation range of fully developed turbulence, Eur. Phys. Let., 24(4), 275-279. 
[21] Benzi, R., Paladin, G., Parisi, G., Vulpiani, A. (1984). Power spectra in two-dimensional turbulence, J. Phys. A.

[22] Beylkin, G. (1991). Wavelets, MRA and fast numerical algorithms, in INRIA lectures.

[23] Beylkin, G., Coifman, R., and V. Rokhlin, (1991). Fast wavelet transforms and numerical algorithms I, Comm. Pure Appl. Math., 43, 141-183.

[24] Bertoluzza, S. (1995). Adaptive wavelet collocation method for the solution of Burgers equation, Trans. Th. Stat. Phys., In the press.

[25] Bertoluzza, S., Maday, Y., and Ravel, J. C. (1994). A dynamically adaptive wavelet method for solving partial differential equations, Comput. Meth. Appl. Mech. Eng., 116, 293-299.

[26] Bertoluzza, S., Naldi, G., and Ravel, J. C. (1994). Wavelet methods for the numerical solution of boundary value problems on the interval, Wavelets: Theory Algorithms and Applications, C. K. Chui, L. Montefusco and Puccio eds, (Academic Press).

[27] Bockhorn, H., Fröhlich, J. and Schneider, K. (1996). An adaptive two-dimensional Wavelet-Vaguelette Algorithm for circular flames, Preprint Universität Karlsruhe, Institut für Chemische Technik.

[28] Bockhorn, H., Fröhlich, J. and Schneider, K. (1996). Direkte numerische Simulation turbulenter reaktiver Strömungen, Forschungszentrum Karlsruhe, Wissenschaftliche Berichte, In the press.

[29] Boussinesq, J. (1877). Essai sur la théorie des eaux courantes, Mémoire de l'Acad. Sci. Paris, 23(1), 1-680.

[30] Brandt, A. (1984). Multigrid techniques with applications to fluid dynamics: 1984 guide, VKI Lecture Series, 1-176.

[31] Cadot, O., Douady, S., and Couder, Y. (1995). Characterization of the low-pressure filaments in a three-dimensional shear flow, Phys. Fluids, 7(3), 630-646.

[32] Cafarelli, L., Kohn, R., and Nirenberg, L. (1982). Partial regularity of suitable weak solutions of the Navier-Stokes equations, Comm. Pure Appl. Math., 35, 771-831.

[33] Caglioti, E., Lions, P. L., Marchioro, C., and Pulvirenti, M. (1992). A special case pf stationary flows for two-dimensional Euler equations: a statistical mechanics description, Comm. Math. Phys., 143, 501-525.

[34] Cannone, M. (1995). Ondelettes, paraproduits et Navier-Stokes, (Diderot Editeur).

[35] Castaing, B. (1989). Consequences d'un principe d'extremum en turbulence, J. Physique, 59, 147-156.

[36] Castaing, B., Gagne, Y., and Hopfinger, E. (1990). Velocity probability density functions of high reynolds number turbulence, Physica D, 46, 177-200.

[37] Cantwell, B. J. (1981). Organized motion in turbulent flow, Ann. Rev. Fluid Mech., 13, 457-515.

[38] Charton, P. (1996). Produits de matrices rapides en bases d'ondelettes : application à la résolution numérique d'équations aux dérivées partielles", Thèse de doctorat de l'Université Paris 13.

[39] Charton, P., and Perrier, V. (1995). Factorisation sur bases d'ondelettes du noyau de la chaleur et algorithmes matriciels rapides associés, $C$. R. Acad. Sci. Paris, série I, 320, 1013-1018.

[40] Charton, P. and Perrier, V. (1996). A pseudo-wavelet scheme for the 
two-dimensional Navier-Stokes equation. Comp. Appl. Math. 15, 139-160.

[41] Chasnov, J. K. (1997). On the decay of two-dimensional homogeneous turbulence. Phys. Fluids, 9(1), 171-180.

[42] Cohen, A., Daubechies, I., and Vial, P. (1993). Wavelets on the interval and fast wavelet transforms, Appl. Comp. Harm. Anal., 1, 157-188.

[43] Couder, Y., Douady, S., and Cadot, O. (1994). Vorticity filaments, Turbulence: a Tentative Dictionary, ed. Tabeling and Cardoso, NATO ASI Series, Plenum Press.

[44] Dahmen, W., Prössdorf, S., and Schneider, R. (1994). Multiscale methods for pseudo-differential equations on smooth closed manifolds, Wavelets: Theory Algorithms and Applications, C. K. Chui, L. Montefusco and Puccio eds, Academic Press.

[45] Davis, A., Marshak, A. and Wiscombe, W. (1994). Wavelet-based multifractal analysis of non-stationary and/or intermittent geophysical signals, in: Wavelet Transforms in Geophysics, eds. E. Foufoula-Georgiou and P. Kumar, 249-298.

[46] Desnjanski, V., and Novikov, E.A. (1974). Model of cascade processes in turbulent flows, Appl. Math. Mech., 38(3), 507-513.

[47] DeVore, R., Jawerth, B., and Popov, V. (1992). Compression of wavelet decomposition, Am. J. Math., 114, 737-785.

[48] Domaradzki, J. A. (1992). Nonlocal triad interactions and the dissipation range of isotropic turbulence, Phys. Fluids A, 4, 2037.

[49] Domaradzki, J. A., and Rogallo, R. S. (1990). Local energy transfer and nonlocal interactions in homogeneous, isotropic turbulence, Phys. Fluids A, 2, 413 .

[50] Domaradzki, J. A., Rogallo, R. S. and Wray, A. A. (1990). Interscale energy transfer in numerically simulated turbulence, CTR, Proceedings of the Summer Program.

[51] Donoho, D. L. (1992). Interpolating Wavelet Transforms. Preprint Stanford University.

[52] Donoho, D. (1993). Unconditional bases are optimal bases for data compression and statistical estimation, Appl. Comp. Harm. Anal., 1, n. 1, 100-115.

[53] Dupree, T. (1992). Coarse-grain entropy in two-dimensional turbulence, Phys. Fluid B, 10, 3101-3114

[54] Dryden, H. (1948). Recent advances in the mechanics of boundary layer flow, Advances in Applied Mechanics, 1, 1-40, (Academic Press).

[55] Duval-Destin, M., and Murenzi, R. (1993). Spatio-temporal wavelets: application to the analysis of moving patterns, Progress in Wavelet Analysis and Applications, ed. Meyer and Roques, (Editions Frontières), 399-408.

[56] Einstein, A. (1914). Méthode pour la détermination de valeurs statistiques d'observations concernant des grandeurs soumises à des fluctuations irrégulières, Archive des Sciences Physiques et Naturelles, 37, 254-255.

[57] Elhmaidi, D., Provenzale, A., and Babiano, A. (1993). Elementary topology of two-dimensional turbulence from a Lagrangian viewpoint and single-particle dispersion, J. Fluid Mech., 257, 533-558.

[58] Elliott, F. W., and Majda, A. J. (1994). A wavelet Monte-Carlo method for turbulent diffusion with many spatial scales, J. Comput. Phys., 113(1), $82-111$. 
[59] Elliott, F. W., and Majda, A. J. (1995). A new algorithm with plane waves and wavelets for random velocity fields with many spatial scales, J. Comput. Phys., 117(1), 146-162.

[60] Elliott, F. W. and Majda, A. J. 1996 Pair dispersion over an inertial range spanning many decades. Phys. Fluids 8(4), 1052-1060.

[61] Eyink, G.L., and Spohn, H. (1993). Negative-temperature states and large-scale, long-lived vortices in two-dimensional turbulence, J. Stat. Phys., 70, n. 3/4, 833-887.

[62] Eyink, G.L. (1995). Space-scale locality and semi-Lagrangian wavelets (energy dissipation without viscosity in ideal hydrodynamics), Preprint, Physics Department, University of Illinois at Urbana-Champain.

[63] Farge, M. (1979). Notes sur la turbulence. Rapport pour le cours de J. M. Levy-Leblond, Université Paris VII.

[64] Farge, M. (1992). Wavelet Transforms and their Applications to Turbulence, Ann. Rev. Fluid Mech., 24, 395-457.

[65] Farge, M. (1992). The continuous wavelet transform of two-dimensional turbulent flows, Wavelets and their Applications, ed. Ruskai et al., (Jones and Bartlett), 275-302.

[66] Farge, M. (1994). Wavelets and two-dimensional turbulence, Computational Fluid Dynamics, 1, (John Wiley \& Sons), 1-23.

[67] Farge, M., Goirand, E., Meyer, Y., Pascal, F. and Wickerhauser, M. V. (1992). Improved predictability of two-dimensional turbulent flows using wavelet packet compression, Fluid Dyn. Res., 10, 229-250.

[68] Farge, M., Guezennec, Y., Ho, C. M., and Meneveau, C. (1990). Continuous wavelet analysis of coherent structures, Proceedings of the Summer Program of the CTR, NASA-Ames and Stanford University, $331-348$.

[69] Farge, M. and Holschneider, M. (1990). Interpretation of two-dimensional turbulence spectrum in terms of singularity in the vortex cores, Europhys. Lett., 15(7), 737-743.

[70] Farge, M., Hunt, J. C. R., and Vassilicos, J. C. (1993). Wavelets, fractals and fourier transforms, (Clarendon Press).

[71] Farge M., Kevlahan N., Perrier V. and Goirand E., (1996). Wavelets and Turbulence, Proc. IEEE, 84, No. 4, p. 639-669.

[72] Farge M., and Philipovitch, T. (1993). Coherent structure analysis and extraction using wavelets, in Progress in Wavelet Analysis and Applications, (ed. Y. Meyer and S. Roques), (Editions Frontières), 477-481.

[73] Farge, M. and Rabreau, G. (1988). Transformée en ondelettes pour détecter et analyser les structures cohérentes dans les écoulements turbulents bidimensionels. C. R. Acad. Sci. Paris Ser. II 307, 433-462.

[74] Farge, M. and Schneider, K. (1997). Wavelet forcing for numerical simulation of two-dimensional turbulence. submitted to C. R. Acad. Sci. Paris Série II.

[75] Federbush, P. (1993). Navier and stokes meet the wavelet, Comm. Math. Phys., 155, 219-248.

[76] Fournier, A. (1995). Wavelet representation of lower atmospheric long nonlinear wave dynamics governed by the Benjamin-Davis-Ono-Burgers equation, in Proceedings SPIE: Wavelet Applications II, (ed. H. H. Szu), 2491, 672-687.

[77] Frick, P. (1993). Choix des ondelettes pour les modèles hiérarchiques de la 
turbulence, Progress in Wavelet Analysis and Applications, (ed. Y. Meyer and S. Roques), (Editions Frontières), 483-490.

[78] Frick, P., Dubrulle, P., and Babiano, A. (1995). Scale invariance in a class of shell models, Phys. Rev. E, 51(5).

[79] Frick, P., and Zimin, V. (1993). Hierarchical models of turbulence, Wavelets, Fractals and Fourier Transforms, ed. Farge, Hunt and Vassilicos, (Clarendon Press), 265-283.

[80] Frisch, U., Sulem, P. L. and Nelkin, M. (1978). A simple dynamical model of intermittent fully developed turbulence, J. Fluid Mech., 87, 719-736

[81] Frisch, U., and Vergassola, M. (1990). A prediction of the multifractal model: the intermediate dissipation range, Europhys. Lett. 14(5), 439-444.

[82] Fröhlich, J. and Schneider, K. (1995). A Fast Algorithm for Lacunary Wavelet Bases related to the Solution of PDEs, C. R. Math. Rep. Acad. Sci. Canada, Vol. XVII, no. 6, 283-286.

[83] Fröhlich, J. and K. Schneider, K. (1994). An adaptive wavelet Galerkin algorithm for one and two dimensional flame computation, Eur. J. Mech. B/Fluids, 13(4), 439-471.

[84] Fröhlich, J. and Schneider, K. (1997). An Adaptive Wavelet-Vaguelette Algorithm for the Solution of PDEs, J. Comput. Phys., 130, 174-190.

[85] Fröhlich, J. and Schneider, K. (1996). Numerical simulation of decaying turbulence in an adaptive wavelet basis, Appl. Comput. Harm. Anal., 3, 393-397.

[86] Fröhlich, J. and Schneider, K. (1997). On the discretization of the convection term in an adaptive wavelet algorithm for PDEs, Preprint, Universität Karlsruhe.

[87] Gagne, Y. (1987). Etude expérimentale de l'intermittence et des singularités dans le plan complexe en turbulence développée. Thesis, Université de Grenoble, France.

[88] Gagnon, L. Lina, J. M. (1994). Symmetric Daubechies' wavelets and mumerical solution of the non-linear Schrödinger's equation, Rapport techniaue, Laboratoire de Physique Nucléaire, Université de Montréal, n. 16, 1994.

[89] Gilbert, A. D. (1988). Spiral structures and spectra in 2-D turbulence, J. Fluid Mech., 193, 475-497.

[90] Glowinski, R., Lawton, W. M., Ravachol, M. and Tenenbaum, E. (1990). Wavelet solution of linear and nonlinear elliptic parabolic and hyperbolic problems in one space dimension, Proceedings of the 9th International Conference on Numerical Methods in Applied Sciences and Engineering, SIAM, Philadelphia.

[91] Gottlieb, D. and Orszag, S.A. (1977). Numerical analysis of spectral methods: theory and applications, Regional Conference Series, SIAM-CBMS, Philadelphia.

[92] Harten, A. (1994). Adaptive multiresolution schemes for shock computations, J. Comput. Phys., 115, 319-338.

[93] Holschneider, M., and Tchamitchian, P. (1991). Pointwise analysis of Riemann's 'non-differentiable' function, Inventiones Mathematicae, 105, $157-176$.

[94] Hunt, J. C. R., and Vassilicos, J. C. (1991). Kolmogorov's contributions to the physical and geometrical understanding of small-scale turbulence and recent developments, Proc. R. Soc. Lond. A, 434, 183-210. 
[95] Jaffard, S. (1994). Some mathematical results about multifractal formalism for functions, in: Wavelets: Theory, Algorithms, and Applications, eds. C. K. Chui, L. Montefusco and L. Puccio, 1-37.

[96] Jaffard, S. (1992). Wavelet methods for fast resolution of elliptic problems, SIAM J. Numer. Anal., 29, 965-987.

[97] Jaffard, S. (1991). Pointwise smoothness, two-microlocalization and wavelet coefficients, Publicacions Matematiques, vol.35, pp. 155-168, 1991.

[98] Jimenez, J. (1981). The role of coherent structures in modelling turbulence and mixing, Springer.

[99] Joyce, G. and Montgomery, D. (1973). Negative temperature states for the two-dimensional guiding-center plasma, J. Plasma Phys., 10, 107.

[100] Kevlahan, N. K.-R. and Farge, M. 1997 Vorticity filaments in two-dimensional turbulence: creation, stability and effect. J. Fluid Mech. In the press.

[101] Kevlahan, N. K.-R. and Vassilicos, J. C. (1994). The space and scale dependencies of the self-similar structure of turbulence, Proc. R. Soc. Lond. A., 447, 341-363.

[102] Kline, S. J., Reynolds, W. C. Schraub, F. A. and Rundstadler, P.W. (1967). The structure of turbulent boundary layer, J. Fluid Mech., 30, 741.

[103] Kolmogorov, A. N. (1941). The local structure of turbulence in incompressible viscous fluid for very large reynolds numbers, $C$. R. Acad. Sci. USSR, 30, 301-305.

[104] Kolmogorov, A. N. (1941). On degeneration of isotropic turbulence in an incompressible viscous liquid, C. R. Acad. Sci. USSR, 31, 538-540.

[105] Kolmogorov, A. N. (1941). Dissipation of energy in the locally isotropic turbulence, C. R. Acad. Sci. USSR, 32, 16-18.

[106] Kolmogorov, A. N. (1962). A refinement of previous hypotheses concerning the local structure of turbulence in a viscous incompressible fluid at high Reynolds number, J. Fluid Mech., 13, 82-85.

[107] Kraichnan, R. H. (1967). Inertial ranges in two-dimensional turbulence, Phys. Fluid, 10, 1417-1423.

[108] Kraichnan, R. H. and Montgomery, D. (1982). Two-dimensional turbulence, Reports on Progress in Physics, 45, 547.

[109] Latto, A. and Tenenbaum, E. (1990). Compactly supported wavelets and the numerical solution of Burger's equation, C. R. Acad. Sci. Paris, Série I, 311, 903-909.

[110] Lazaar, S., Liandrat, J. and Tchamitchian, P. (1994). Algorithme à base d'ondelettes pour la résolution numérique d'équations aux dérivées partielles à coefficients variables, C. R. Acad. Sci. Paris, série I, 319, 1101-1107.

[111] Lazaar, S., Ponenti, P. J. Liandrat, J. and Tchamitchian, P. (1994). Wavelet algorithms for numerical resolution of partial differential equations, Comput. Methods Appl. Mech. Eng., 116, 309-314.

[112] Legras, B. and Dritschel, D. G. (1993). A comparison of the contour surgery and pseudo-spectral methods, J. Comput. Phys., 104(2), 287-302.

[113] Legras, B., Santangelo, P., and R. Benzi, R. (1988). High-resolution numerical experiments for forced two-dimensional turbulence, Europhys. Lett., 5, 37-42.

[114] Leith, C. E. (1990). Stochastic backscatter in a subgrid-scale model: plane shear mixing layer, Phys. Fluids A 3, 297-299. 
[115] Lemarié-Rieusset, P. G. (1994). Un théorème d'inexistence pour les ondelettes vecteur à divergence nulles, CRAS Série I, 310, 811-813.

[116] Lemarié-Rieusset, P. G. (1992). Analyses multirésolutions non-orthogonales, commutation entre projecteur et derivation, et ondelettes à divergence nulle, Revista Mat. Iberoamerica, 8, 221-236.

[117] Leonard, A. (1974). Adv. Geophys., A 18, 17.

[118] Leonard, A. (1980). Vortex methods for flow simulation, J. Comp. Phys., 37, 289-335.

[119] Lesieur, M. (1996). New trends in large-eddy simulations of turbulence, Ann. Rev. Fluid Mech., 28, 45-82.

[120] Lewalle, J. (1993). Wavelet transforms of the Navier-Stokes equations and the generalized dimensions of turbulence. Appl. Sci. Res. 51, 109-113.

[121] Lewalle, J. (1993). Energy dissipation in the wavelet-transformed Navier-Stokes equations. Phys. Fluids A 5, 1512-1513.

[122] Lewalle, J. (1996). Gaussian wavelets as Green's functions. Phys. Rev. Lett. E. In the press.

[123] Liandrat, L., Perrier, V. and Tchamitchian, P. (1992). Numerical resolution of nonlinear partial differential equations using the wavelet approach, in Wavelet and their applications, Ruskai et al. (ed.), Jones and Barlett, 227-238.

[124] Liepmann, H. W. (1962). Free turbulent flows, Mécanique de la turbulence, ed. Favre, 211-227.

[125] Liepmann, H. (1979). The rise and fall of ideas in turbulence, Scientific American March-April.

[126] Liepmann, H. W. (1997). Private communication.

[127] Mc Williams, J. C. (1984). The emergence of isolated coherent vortices in turbulent flows, J. Fluid Mech., 146, 21-43.

[128] Maday, Y., Perrier, V., and Ravel, J. C. (1991). Adaptativité dynamique sur base d'ondelettes

pour l'approximation d'équations aux dérivées partielles, C. R. Acad. Sci. Paris, série I, 312, 405-410.

[129] Mallat, S. and Hwang, W. H. (1992). Singularity detection and processing with wavelets, IEEE Trans. Inform. Theory, 38, 617-643.

[130] Mandelbrot, B. (1975). Intermittent turbulence in self-similar cascades: divergence of high moments and dimension of carrier, J. Fluid Mech., 62, $331-358$.

[131] Marchioro, C. and Pulvirenti, M. (1994). Mathematical Theory of incompressible nonviscous fluids, (Springer).

[132] Marion, M. and R. Temam, R. (1989). Nonlinear Galerkin Methods, SIAM J. Num. Anal., 26, 1139-1157.

[133] Meneveau, C. (1991). Analysis of turbulence in the orthonormal wavelet representation, J. Fluid Mech., 232, 469-520.

[134] Meyer, Y. (1990). Ondelettes et opérateurs, Hermann.

[135] Miller, J. (1990). Statistical Mechanics of Euler Equations in Two Dimensions, Phys. Rev. Lett., 65, n. 17, 2137-2140.

[136] Min, I. A., Mezić, I. and Leonard, A. (1996). Lévy stable distributions for velocity and velocity difference in systems of vortex elements. Phys. Fluids 8(5), 1169-1180.

[137] Moffatt, H. K. (1984). Simple topological aspects of turbulent velocity dynamics, in Proc. IUTAM Symp. on turbulence and chaotic phenomena in 
fluids (ed. T. Tatsumi), 223, (Elsevier).

[138] Mohammadi, B. and Pirroneau, O. (1994). Analysis of the $k$ - $\epsilon$ Turbulence Model, (Wiley-Masson).

[139] Monasse, P. and Perrier, V. (1995). Ondelettes sur l'intervalle pour la prise en compte de conditions aux limites, C. R. Acad. Sci. Paris, 321, Série I, 1163-1169.

[140] Monasse, P. and Perrier, V. (1995). Orthonormal wavelet bases adapted for partial differential equations with boundary conditions, Prépublications Mathématiques de l'Université Paris-Nord n.95-11.

[141] Monin, A. S. and Yaglom, A. M. (1975). Statistical Fluid Mechanics: Mechanics of Turbulence, (The M.I.T. Press).

[142] Myers, M., Holmes, P., Elezgaray, J. and Berkooz, G. (1995). Wavelet projections of the Kuramoto-Sivashinsky equation I: heteroclinic cycles and modulated waves for short systems, Physica D, 86, 396-427.

[143] Neumann, J. von (1949) Recent theories of turbulence. In Collected works (1949-1963) 6, 437-472, (ed. A. H. Taub). Pergamon Press, (1963).

[144] Obukhov, A. M. (1941). Energy distribution in the spectrum of a turbulent flow, Izv. AN URSS, 4, 453-466.

[145] Onsager, L. (1945). The distribution of energy in turbulence, Phys. Rev., 68, 286.

[146] Onsager, L. (1949). Statistical hydrodynamics, Suppl. Nuovo Cimento, suppl. 6, 279-287.

[147] Parisi, G. and Frisch, U. (1985). Fully developed turbulence and intermittency, Turbulence and Predictability in Geophysical Fluid Dynamics and Climate Dynamics, ed. Ghil, Benzi and Parisi, (North-Holland), 71-88.

[148] Perrier, V. and Basdevant, C. (1989). Periodical wavelet analysis, a tool for inhomogeneous field investigation. Theory and algorithms, Rech. Aérosp. n 1989-3.

[149] Perrier, V., Philipovitch, T. and Basdevant, C. (1995). Wavelet spectra compared to Fourier spectra, J. Math. Phys., 36(3), 1506-1519.

[150] Perrier, V. and Wickerhauser, M. V. (1995). Multiplication of short wavelet series using connection coefficients, Preprint.

[151] Philipovitch, T. (1994). Applications de la transformée en ondelettes continue à la turbulence homogène isotrope bidimensionelle, Thèse de Doctorat, Université Paris VI.

[152] Piomelli, U. Cabot, W. H. Moin, P. and Lee, S. (1990). Subgrid-scale backscatter in transitional and turbulent flows, CTR, Proceedings of the Summer Program, 19-30.

[153] Poliakov, A. M. (1993). Conformal Turbulence, Nucl. Phys. B, 396, 367.

[154] Ponenti, P. (1994). Algorithmes en ondelettes pour la résolution d'équations aux dérivées partielles, Thèse de Doctorat, Université d'Aix-Marseille I.

[155] Qian, S. and Weiss, J. (1993). Wavelets and the numerical solution of Partial Differential Equations, J. Comp. Phys., 106, 155-175.

[156] Reynolds, O. (1883). An experimental investigation of the circumstances which determine whether the motion of water shall be direct and sinuous, and the law of resistance in parallel channels. Phil. Trans. Roy. Soc., $51-105$.

[157] Reynolds, O. (1894). On the dynamical theory of incompressible viscous fluids and the determination of the criterion, Phil. Trans. Roy. Soc. Lond., 
186, 123-164.

[158] Reynolds, W. C. (1976). Computation of turbulent flows, Ann. Rev. Fluid Mech., 8 .

[159] Richardson, L. F. (1922). Weather prediction by numerical process, (Cambridge University Press, Cambridge).

[160] Richardson, L. F. and Gaunt, J. A. (1993). Diffusion regarded as a compensation for smoothing, Memoirs of the Royal Meteorological Society, 3, no. 30, 171-175, 1930 (Reprinted in the Collected papers of Lewis Fry Richardson, 1, (Cambridge University Press), 773-777).

[161] Robert, R. (1990). Etat d'équilibre Statistique pour l'écoulement bidimensionnel d'un fluide parfait, C. R. Acad. Sci. Paris, Série I, 311, $575-578$

[162] Robert, R. (1991). A maximum-entropy principle for two-dimensional perfect fluid dynamics, J. Stat. Phys., 65, 531-553.

[163] Robert, R. and Sommeria, J. (1991). Statistical equilibrium states for two-dimensional flows, J. Fluid Mech., 229, 291-310.

[164] Roshko, A. (1961). J. Fluid Mech., 10, 345.

[165] Saffman, P. G. (1971). A note on the spectrum and decay of random two-dimensional vorticity distribution, Stud. Appl. Math., 50, 377-383.

[166] Schneider, K., Kevlahan, N. and Farge, M., (1997). Preprint Universität Karlsruhe, Institut für Chemische Technik.

[167] Sommeria, J. Staquet, C. and Robert, R. (1991). Final equilibrium state of a two-dimensional shear layer, J. Fluid Mech., 223, 661-689.

[168] Taylor, G. I. (1921). Diffusion by continuous movements, Proc. Lond. Math. Soc. Ser. 2, 20, 196-211.

[169] Taylor, G. I. (1935). Statistical theory of turbulence, Proc. Roy. Soc. Lond. A, 151, 421-478.

[170] Taylor, G. I. (1938). The spectrum of turbulence. Proc. Roy. Soc. Lond. A 164, 476-490.

[171] Tennekes, H. and Lumley, J. L. (1972). A first course in turbulence, (MIT Press).

[172] Tong, P. and Goldburg, W. I. (1988). Experimental study of relative velocity fluctuations in turbulence. Phys. Lett. A 127, 147.

[173] Townsend, A. A. (1956). The structure of turbulent shear flow, (Cambridge University Press).

[174] Urban, K. (1994). A wavelet-Galerkin algorithm for the driven-cavity-Stokes-problem in two space dimensions, R.W.T.H. Aachen preprint.

[175] Van Atta, C. W. and Antonia, R. A. (1980). Reynolds dependence of sknewness and flatness factors of turbulent velocity derivatives, Phys. Fluids, 23, 252-257.

[176] Vassilicos, J. C. (1992). The multi-spiral model of turbulence and intermittency, Topological Aspects of the Dynamics of Fluids and Plasmas, Kluwer, 427-442.

[177] van der Water, W. (1993). Experimental study of scaling in fully developed turbulence, Turbulence in Spatially Extended Systems, ed. Benzi, Basdevant and Ciliberto, 189-213.

[178] Vergassola M. and Frisch, U. (1991). Wavelet transforms of self-similar processes, Physica D. 54, 58-64.

[179] Walter, G. (1992). A sampling theorem for wavelet subspaces. IEEE Trans. 
Inform. Theory, 38, 881 .

[180] Weiss, J. (1992). The dynamics of enstrophy transfer in two-dimensional hydrodynamics, Physica D, 48, 273.

[181] Weiss, J. B. and McWilliams, J. C. (1991). Non-ergodicity of point vortices. Phys. Fluids A 3, 835.

[182] Wickerhauser, M. V., Farge, M., Goirand, E. Wesfreid, E. and Cubillo, E. (1994). Efficiency comparison of wavelet packet and adapted local cosine bases for compression of a two-dimensional turbulent flow, Wavelets: Theory, Algorithms and Applications, ed. Chui, Montefusco and Puccio, Academic Press, 509-531.

[183] Yaglom, A. M. (1986). Einstein's work on methods of processing fluctuating series of observations and the role of these methods in meteorology, Izv. Atmos. Ocean. Phys., 22, 1.

[184] Yakhot, V. and Orszag, S. A. (1986). Phys. Rev. Lett., 57, 1722.

[185] Zabusky, N. (1984). Computational synergetics. Phys. Today July, 2-11.

[186] Zimin, V. (1981). Hierarchical model of turbulence, Izv. Atmos. Ocean Phys., 17, 941-949.

[187] Zimin, V. (1995). Wavelet based model for small-scale turbulence, Phys. Fluids, 7 (12), 2925-2927, 1995.

[188] Zygmund, A. (1959). Trigonometric series, (Cambridge University Press, Cambridge). 